\section{Kidney \\ Blood Pressure Research}

\title{
Urine Proteomics Revealed a Significant Correlation Between Urine-Fibronectin Abundance and Estimated-GFR Decline in Patients with Bardet-Biedl Syndrome
}

\author{
Marianna Caterino ${ }^{a, b} \quad$ Miriam Zacchia $^{d}$ Michele Costanzo $^{a, b, c} \quad$ Giuliana Bruno $^{e}$ \\ Davide Arcaniolo ${ }^{f}$ Francesco Trepiccione $^{d}$ Rosa Anna Sicilianog \\ Maria Fiorella Mazzeog Margherita Ruoppolo ${ }^{a, b, c} \quad$ Giovambattista Capasso $^{d}$ \\ aDipartimento di Medicina Molecolare e Biotecnologie Mediche, Università degli Studi di Napoli, \\ "Federico II", Naples, 'bEINGE Biotecnologie Avanzate, Naples, 'Associazione culturale DiSciMuS, \\ Casoria, Naples, 'Division of Nephrology, Department of Cardio-thoracic and Respiratory Sciences, \\ University of Campania "Luigi Vanvitelli", Naples, eIRGS Biogem, Via Camporeale, Ariano Irpino, Avellino, \\ fUrology Unit, University of Campania "Luigi Vanvitelli", Naples, IIstituto di Scienze dell' Alimentazione, \\ CNR Avellino, Italy
}

\section{Key Words}

Bardet-Biedl Syndrome • Kidney dysfunction • Urine proteome • Fibronectin • GFR decline

\begin{abstract}
Background:/Aims: Renal disease is a common cause of morbidity in patients with Bardet-Biedl syndrome (BBS), however the severity of kidney dysfunction is highly variable. To date, there is little information on the pathogenesis, the risk and predictor factors for poor renal outcome in this setting. The present study aims to analyze the spectrum of urinary proteins in BBS patients, in order to potentially identify 1 ) disease-specific proteomic profiles that may differentiate the patients from normal subjects; 2) urinary markers of renal dysfunction. Methods: Fourteen individuals (7 males and 7 females) with a clinical diagnosis of BBS have been selected in this study. A pool of 10 aged-matched males and 10 aged-matched females have been used as controls for proteomic analysis. The glomerular filtration rate (eGFR) has been estimated using the CKD-EPI formula. Variability of eGFR has been retrospectively assessed calculating average annual eGFR decline ( $\triangle \mathrm{eGFR}$ ) in a mean follow-up period of 4 years (3-7). Results: 42 proteins were significantly over- or under-represented in BBS patients compared with controls; the majority of these proteins are involved in fibrosis, cell adhesion and extracellular matrix organization. Statistic studies revealed a significant correlation between urine fibronectin ( $\mathrm{u}-$ FN) $\left(r^{2}=0.28 ; p<0.05\right), C D 44$ antigen $\left(r^{2}=0.35 ; p<0.03\right)$ and lysosomal alfa glucosidase $\left(r^{2} 0.27 ;\right.$
\end{abstract}

M. Caterino and M. Zacchia contributed equally to this paper.

\begin{tabular}{ll}
\hline Miriam Zacchia & Division of Nephrology, Department of Cardio-Thoracic and Respiratory Sciences, University of Campania \\
& "Luigi Vanvitelli", via Pansini n. 5, Policlinico Nuovo, 80131, Naples (Italy) \\
& Tel. +0039-0815666650 E-Mail miriamzacchia@virgilio.it
\end{tabular} 


\section{Kidney Blood Pressure Research}

$p<0.05)$ abundance with the eGFR. In addition, $u-F N\left(r^{2}=0.2389 ; p<0.05\right)$ was significantly correlated with $\triangle \mathrm{eGFR}$. Conclusion: The present study demonstrates that urine proteome of BBS patients differs from that of normal subjects; in addition, kidney dysfunction correlated with urine abundance of known markers of renal fibrosis.

\section{Introduction}

Kidney dysfunction is a well-known clinical feature of BBS patients; however, its pathogenesis is still largely unknown. The syndrome has a genetic origin, and to date 21 genes have been associated with human BBS but it is presumable that other unknown genes are yet to be discovered [1]. Even though many efforts have been devoted to address genotype-phenotype correlation, there is still little information on this issue [2-4].

Renal abnormalities in BBS patients are both structural and functional. The majority of BBS patients show structural defects of the kidney and the urinary tract [5]; kidney dysfunction ranges from renal hyposthenuria to the end stage renal disease (ESRD) [6-9]. Whether the defective ability to concentrate the urine is an early marker of kidney dysfunction is currently unknown [10-13]. The molecular basis of kidney dysfunction is poorly understood. The BBS genes encode proteins that are mainly localized to the centrosome and regulate the function of the primary cilium (PC) [14]. Several studies have linked the disruption of cilia homeostasis with defects in renal development and cysts formation, thus BBS is considered the result of ciliary dysfunction and is included in the group of diseases named ciliopathies. Mouse models of BBS have shown that BBS2, BBS4 and BBS10 depletion lead to systemic-related renal disease $[15,16]$. In addition, in vitro studies have shown that $B B S 10$ is implicated in the mechanisms mediating water re-absorption along the collecting duct (CD) $[17,18]$. The role of BBS genes in human kidney disease is largely unknown. In the recent years, urinary proteomic investigation has been applied to clinical medicine, allowing identification of up- or down-regulated proteins, that may help to clarify the pathophysiology of kidney dysfunction and to discover disease biomarkers [19]. The present study is the first investigation on urine proteome in BBS patients. Our results demonstrate that these patients have a unique urine protein profile. In addition, the correlation between altered proteins and kidney dysfunction may shed light in understanding the patho-physiology of renal disease and may help to predict renal outcome.

\section{Materials and Methods}

\section{Participants}

This study was performed in adherence to the Declaration of Helsinki and was approved by Institution Review Board. All patients or their legal representatives gave their written informed consent. A group of 14 adult individuals with a clinical diagnosis of BBS according to Beales criteria [5] were enrolled in the study. Retinal degeneration was assessed by ophthalmological testings. Obesity was defined as body mass index over $30 \mathrm{~kg} / \mathrm{m}^{2}$. Learning disabilities included defects in writing, reading, speaking, besides deficits of memory and coordination. The patients underwent biochemical analyses to address renal function; in addition, kidney ultrasound was performed. The GFR was estimated with CKD-EPI formula using standardized serum creatinine measurement, a procedure of creatinine testing calibrated to a traceable reference method (Isotope Dilution Mass Spectrometry or IDMS). Mean annual decline in GFR was estimated by the ratio between retrospective data indicating changes in GFR and follow-up duration (recent eGFR-eGFR at initial visit)/follow-up duration (years). Mean follow-up period was 4 years (3-7 years).

All patients underwent a screening for diabetes mellitus by measuring fasting serum glucose (FPG) and glycated hemoglobin (HbA1c). A repeated FPG $\geq 126 \mathrm{mg} / \mathrm{dl}$ (7.0 mmol/l) and/or HbA1c levels of $6.5 \%$ or greater were considered diagnostic of diabetes. Hypertension was defined as a systolic blood pressure of $140 \mathrm{~mm} \mathrm{Hg}$ or more, or a diastolic blood pressure of $90 \mathrm{~mm} \mathrm{Hg}$ or more. 


\section{Kidney Blood Pressure Research}

Patients with an eGFR lower than $55 \mathrm{~mL} / \mathrm{min} / 1.73 \mathrm{~m}^{2}$, with chronic infection and/or malignancy were excluded. Twenty healthy volunteers, age- and gender-matched (ten females and ten males) were used as controls.

\section{Sample collection and preparation}

Urine samples were collected as described [20]. After overnight fasting, the second void urine sample was collected from each patient and control and immediately centrifuged at $2000 \mathrm{rpm}$ for $10 \mathrm{~min}$. Urine creatinine concentration was measured by the analytical BM/Hitachi 904 as described. Urine samples from healthy volunteers were pooled in order to obtain two gender-specific control groups, composed of equal urine volumes from each subject. Urinary creatinine concentration was measured in samples from each single control-subject and in pooled controls. Small molecules and salts were removed by ultra-filtration and subsequent precipitation steps.

Samples were 50-fold-concentrated using a 3kDa cut-off ultrafiltration unit (3000 NMWL, Amicon ${ }^{\circledR}$ Ultra-15 Centrifugal Filters, Merck Millipore, Tulla green Carrigtwohill, Co. Cork, Ireland) at 3000 rpm for 60 $\min$. The recovered urine was precipitated adding cold acetone and stored at $-20^{\circ} \mathrm{C}$ overnight. Pellets were then separated from supernatant, air-dried and re-suspended in a buffer solution consisting of 7M Urea, $2 \mathrm{M}$ Thiourea, 4\% Chaps, 30mM Tris-HCl pH=7, 8. Two independent 10\% SDS-preparative gel $(16 \times 20 \mathrm{~cm})$ were used for female and male groups, respectively. Protein extracts from patients and control pools were resolved on a 10\% 1D-SDS-polyacrylamide gel and stained as previously described [21, 22]. Each lane was cut, digested with trypsin and analyzed by LC-MS/MS [23, 24].

\section{LC-MS/MS analysis, proteins identification and quantization}

The peptide mixtures were analyzed by LC-MS/MS using a Q-ExactiveTM (ThermoScientific, Bremen, Germany) coupled to an UltiMate 3000 RSLCnano LC system (ThermoScientific), including a nano-pumps binary system and a third low-pressure micro-pump. The peptide mixtures were concentrated on a trapping pre-column (Acclaim PepMap C18, 300 $\mathrm{m} \times 5$ mm nanoViper, $5 \mu \mathrm{m}, 100 \AA$ A, Thermo Scientific), using 0.05\% formic acid and $2 \%$ acetonitrile. The peptide separation was performed using a C18 column (Acclaim Easy Spray PepMap RSLC C18, $75 \mu \mathrm{m} \times 15 \mathrm{~cm}$ nanoViper, $3 \mu \mathrm{m}, 100 \AA$, Thermo Scientific). Peptides were eluted with a nonlinear gradient set as follows: $4 \%$ B for 5 minutes, from 4 to $40 \%$ B in 45 minutes and from 40 to $90 \%$ B in 1 minute at flow rate of $300 \mathrm{~nL} / \mathrm{min}$. A and B mobile phases were $0.1 \%$ formic acid and $80 \%$ acetonitrile, $0,08 \%$ formic acid respectively. The mass spectrometer Q-ExactiveTM was setup in a data dependent Full MS/ddMS2, enabling the acquisition of MS/MS spectra for the ten most intense precursor ions (top ten) and dynamic exclusion of $30 \mathrm{sec}$. Resolution was set to 70000 for MS spectra acquisition and 17500 for MS/MS spectra acquisition.

Proteomic analysis was performed using ThermoProteomeDiscoverer ${ }^{\mathrm{TM}}$ platform (version 2.1.0.81; ThermoScientific), interfaced to the Sequest HT Search Engine server (University of Washington, United States) for protein identification [25]. Proteins identified by a minimum of two peptides were accepted. Only proteins identified in at least $70 \%$ of patients (5/7) underwent further analyses. The spectral counting approach was used to compare protein expression profile in patients versus control groups [26, 27]. The relative protein abundance was evaluated by the $\mathrm{SpC} \log$ ratio $\left(\mathrm{R}_{\mathrm{sC}}\right)$ [28]. To ensure the quantitative accuracy, two methods were used. To calculate each protein Normalized Spectral Abundance Factor (NSAF), the number of spectral counts (SpCs) was divided by protein length (SAF) and normalized to the sum of SAFs in a given lane. The NSAF of each ' $i$ ' protein was then normalized against urine creatinine concentration (cNSAFi). The NSAF of each protein from the control pools was normalized by urine creatinine concentration $\left(\mathrm{CNSAF}_{\text {poolfemale }}\right.$ and $\mathrm{CNSAF}_{\text {poolmale }}$ ). Finally, to each 'i' protein the $\mathrm{R}_{\mathrm{NSAF}}$ was calculated as the binary logarithm of a ratio of $\mathrm{cNSAFi}$ versus $\mathrm{CNSAF}_{\text {pool }}$.

\section{Data mining and statistical analysis}

The dataset of differentially represented proteins was analyzed using 'STRING: functional protein association networks' software 7.0 (http://string-db.org/) [29, 30].

The statistical analysis were carried out by means of GrapfPadPrism software. The protein fold changes, expressed as $\mathrm{R}_{\mathrm{NSA}}$, were correlated with eGFR and annual eGFR $(\Delta \mathrm{eGFR}$ ) using Pearson Correlation test. Differences were considered statistically significant for $\mathrm{p}$-values $<0.05$. 


\section{Kidney Blood Pressure Research}

Table 1. Clinical features of female BBS patients. BMI, Body Mass Index; eGFR, Estimated Glomerular Filtration Rate; PCR, urine protein to creatinine ratio

\begin{tabular}{|c|c|c|c|c|c|c|c|}
\hline Description & $\mathrm{X} 1$ & $\mathrm{X} 2$ & $\mathrm{X} 3$ & $\mathrm{X} 4$ & $\mathrm{X} 5$ & $\mathrm{X} 6$ & X7 \\
\hline Age & 43 & 27 & 19 & 19 & 18 & 25 & 22 \\
\hline $\mathrm{BMI}, \mathrm{kg} / \mathrm{m}^{2}$ & 32.3 & 27 & 28.9 & 32 & 29.8 & 42.8 & 30 \\
\hline Retinal degeneration & + & + & + & _- & + & + & + \\
\hline Polydactyly & + & + & + & + & _- & + & _ \\
\hline Learning disabilities & _ & + & + & + & + & _ & _ \\
\hline Renal structural abnormalities & + & + & + & + & - & + & + \\
\hline PCR (mg/g) & 132 & 82 & 87 & 85 & 73 & 45 & 43 \\
\hline eGFR & 87.6 & 72.7 & 111.9 & 130 & 128.6 & 81.2 & 111.3 \\
\hline$\Delta$ eGFR/year & -4.7 & -6.5 & -2.8 & 0.8 & 0.2 & -4.5 & -6 \\
\hline Diabetes & - & - & - & - & _- & + & - \\
\hline Hypertension & & & & & - & & - \\
\hline
\end{tabular}

Table 2. Clinical features of male BBS patients. BMI, Body Mass Index; eGFR, Estimated Glomerular Filtration Rate; PCR, urine protein to creatinine ratio

\begin{tabular}{|c|c|c|c|c|c|c|c|}
\hline Description & Y1 & Y2 & Y3 & $\mathrm{Y} 4$ & Y5 & Y6 & Y7 \\
\hline Age & 29 & 30 & 23 & 27 & 33 & 27 & 25 \\
\hline BMI, $\mathrm{kg} / \mathrm{m}^{2}$ & 23 & 23 & 28 & 31 & 29.1 & 26 & 43,7 \\
\hline Retinal degeneration & + & + & + & + & + & + & + \\
\hline Polydactyly & + & + & _ & _ & + & + & + \\
\hline Learning disabilities & + & + & + & + & + & _ & _ \\
\hline Renal structural abnormalities & + & + & + & _ & _ & _ & + \\
\hline PCR (mg/g) & 72 & 86 & 56 & 55 & 46 & 45 & 550 \\
\hline eGFR & 60.1 & 79.1 & 126.7 & 92.9 & 121.9 & 119.2 & 55.6 \\
\hline$\Delta$ eGFR/year & -10.1 & -3.4 & -2 & -1 & -0.7 & -1.1 & -4.2 \\
\hline Diabetes & _ & _ & _ & _ & - & - & + \\
\hline Hypertension & + & 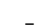 & . & _ & _ & - & + \\
\hline
\end{tabular}

\section{Results}

\section{Cohort}

Fourteen adult BBS patients with clinical diagnosis of BBS have been selected for the study. The main features are listed in Tables 1 and 2. Patients were aged 19-43 years old; all patients with the exception of one had retinal dystrophy; BMI ranged between 23 and $43.7 \mathrm{~kg} / \mathrm{m}^{2}$. Polydactyly and learning disabilities were present in $10 / 14$ and 9/14 patients respectively; diabetes was present in only 1 male and 1 female patient. 2 male patients (and no any female patient) were hypertensive. Renal structural abnormalities on ultrasound were present in 10/14 patients, with calyceal and parenchymal cysts, unilateral renal hypoplasia, ureteric tract malformation being the most common defects. Protein to creatinine ratio (PCR) was over $150 \mathrm{mg} / \mathrm{g}$ in one male patient. The estimated GFR ranged from 55 to 130 $\mathrm{mL} / \mathrm{min} / 1.73 \mathrm{~m}^{2}$. Annual GFR decline varied among subjects; interestingly, the majority of patients showed a progression of kidney disease.

\section{Urine proteome of BBS patients}

Two independent gender comparative analyses were performed. Urine proteomes of 7 female and 7 male BBS subjects were compared with the proteome of the gender-matched control pool. Urinary proteins were resolved on a 10\% 1D-SDS-polyacrylamide gel as shown in Fig. 1. Gel bands were cut and proteins identified by LC-MS/MS. The analysis revealed a dataset constituted by 73 and 66 proteins in female and male patients, respectively. The details of protein identification is reported in supplementary Tables $1 \mathrm{~S}$ and 2S. For all supplemental material see www.karger.com/doi/10.1159/000488096.

Label-free proteomic analysis was performed to compare proteome composition between patients and controls. In order to estimate the differences in urine proteome, the relative abundance of each protein was calculated by two spectral counting parameters, $\mathrm{R}_{\mathrm{SC}}$ and $\mathrm{R}_{\mathrm{NSAF}}$. Female and male datasets are reported in Table 3 and Table 4, respectively. Fig. 2 shows the urine protein abundance (fold changes) based on Rsc and $\mathrm{R}_{\mathrm{NSAF}}$ in female (Fig. 2A) and male (Fig. 2B) BBS individuals. The results were similar in both conditions, confirming the reliability of the quantitative analysis of this study. Female dataset showed 23 


\section{Kidney Blood Pressure Research}

over-represented proteins and 42 underrepresented proteins compared with the control pool. Male dataset showed 27 over-represented proteins and 36 underrepresented proteins compared with the control. 42 proteins were de-regulated in both male and female BBS individuals compared with controls (Table 5). Fig. 3 shows that the majority of these proteins had the same behavior in both female and male BBS; however, some of them showed an opposite abundance in males and females, thus suggesting an inter-gender variability.

Urinary extracellular matrix organization (ECM) proteins and cell adhesion molecules (CAM) were highly deregulated in BBS patients

Protein association networks were analyzed using STRING database (http:// string-db.org/). Proteins were grouped according to the biological and functional connections found by Gene Ontology (GO) Database (Fig. 4). The most representative biological processes were cell adhesion (13 nodes, 0.00513 FDR) and extracellular matrix organization (8 nodes, 0.00776 FDR). The network distributions of the differentially represented BBS urinary proteins revealed the central role of Fibronectin.

Fibronectin (FN), lysosomal alphaglucosidase and CD44 antigen showed a significant correlation with the eGFR in $B B S$ patients

We verified whether urine proteins that were over or under-represented in BBS patients showed any correlation with the eGFR. Statistical analysis revealed that urine FN, CD44 antigen and lysosomal alpha-glucosidase were significantly correlated with the eGFR, as shown in Fig. 5. Interestingly, also vitronectin, IgK chain V, Ig lambda chain V and Isoform 2 of Tyrosine-protein phosphatase non-receptor type substrate 1 (SIRP- $\alpha$ ) were significantly correlated with the eGFR in each group of female and male patients, but not when we considered the cohort in its totality (supplementary Table 3S).

\section{Fibronectin significantly correlated with annual $\triangle e G F R$ in BBS patients}

Urine proteome of BBS patients was correlated with retrospective data indicating the rate of annual change in the eGFR $(\triangle \mathrm{eGFR})$. The rationale of the study was to address whether patients undergoing a faster progression of renal dysfunction may show a specific set of urine proteins. This analysis revealed that urine FN was significantly correlated with the $\triangle \mathrm{eGFR}$. This finding is consistent with the correlation of FN with the eGFR, further supporting the hypothesis that it may be considered as a marker of kidney disease (Fig. 6). 


\section{Kidney \\ Blood Pressure Research}

Caterino et al.: Urine Proteome of Patients with Bardet-Biedl Syndrome

Table 3. Urine protein abundance (fold changes) based on $\mathrm{R}_{\mathrm{SC}}$ and $\mathrm{R}_{\mathrm{NSAF}}$ in female BBS subjects

\begin{tabular}{|c|c|c|c|c|c|c|c|c|c|c|c|c|c|c|c|}
\hline \multirow[b]{2}{*}{ Accession } & \multirow[b]{2}{*}{ Description } & \multicolumn{7}{|c|}{$\mathrm{R}_{\mathrm{sc}}$} & & & & $\mathrm{R}_{\mathrm{NS}}$ & & & \\
\hline & & $\mathrm{x} 1$ & $\mathrm{x} 2$ & $\mathrm{x} 3$ & $\mathrm{x} 4$ & $\mathrm{x} 5$ & $\mathrm{x} 6$ & $\mathrm{x} 7$ & $\mathrm{x} 1$ & $\mathrm{x} 2$ & $\mathrm{x} 3$ & $\mathrm{x} 4$ & $\mathrm{x} 5$ & $\mathrm{x} 6$ & $\mathrm{x} 7$ \\
\hline 000391 & Sulfhydryl oxidase 1 & 2,2 & $-1,5$ & $-1,0$ & $-2,8$ & 1,0 & 0,2 & $-2,0$ & 4,2 & $-0,6$ & 0,0 & $-4,0$ & 1,6 & 0,5 & $-3,4$ \\
\hline 043451 & Maltase-glucoamylase, intestinal & 1,1 & $-1,9$ & $-2,6$ & $-3,1$ & 0,2 & 1,7 & $-2,4$ & 3,1 & $-1,0$ & $-1,8$ & $-4,4$ & 0,8 & 2,1 & $-3,8$ \\
\hline 075594 & peptidoglycan recognition protein 1 & $-5,7$ & $-4,8$ & 2,0 & 0,6 & 0,1 & $-3,6$ & $-0,2$ & $-5,4$ & $-4,9$ & 3,0 & 0,9 & 0,8 & $-3,8$ & $-0,1$ \\
\hline P00734 & Prothrombin & $-0,1$ & 1,1 & 0,0 & $-1,6$ & 0,5 & 0,7 & $-0,4$ & 1,9 & 2,6 & 1,0 & $-1,4$ & 1,2 & 1,0 & $-0,2$ \\
\hline P01620 & Ig kappa chain V-III region SIE & $-1,4$ & $-1,1$ & 0,1 & 2,5 & $-0,5$ & $-4,5$ & $-2,9$ & 0,5 & 0,3 & 1,2 & 2,8 & 0,1 & $-4,7$ & $-3,3$ \\
\hline P01622 & Ig kappa chain V-III region TI & $-3,9$ & $-1,0$ & $-0,4$ & 2,8 & $-1,2$ & $-5,7$ & $-2,6$ & $-2,2$ & 0,4 & 0,7 & 3,1 & $-0,5$ & $-6,9$ & $-2,9$ \\
\hline P01623 & Ig kappa chain V-III region WOL & $-1,5$ & $-1,0$ & 0,1 & 2,5 & $-0,5$ & $-4,5$ & $-3,0$ & 0,5 & 0,5 & 1,2 & 2,8 & 0,1 & $-4,7$ & $-3,3$ \\
\hline P01699 & Ig lambda chain V-I region VOR & 0,5 & $-2,8$ & 1,8 & $-0,1$ & 0,6 & $-1,6$ & $-1,7$ & 2,4 & $-2,9$ & 2,9 & 0,0 & 1,3 & $-1,8$ & $-3,0$ \\
\hline P01834 & Ig kappa chain C region & $-7,3$ & $-3,2$ & $-0,6$ & $-0,9$ & $-3,5$ & $-4,0$ & $-5,3$ & $-7,0$ & $-1,9$ & 0,4 & $-0,6$ & $-2,9$ & $-3,8$ & $-6,7$ \\
\hline P02647 & Apolipoprotein A-I & $-1,3$ & 2,0 & $-2,3$ & 3,5 & 3,2 & 1,6 & 2,4 & $-0,7$ & 3,4 & $-2,6$ & 3,9 & 4,1 & 1,9 & 2,3 \\
\hline P02751 & Fibronectin & 0,7 & 0,1 & $-2,7$ & $-0,2$ & $-0,8$ & $-1,4$ & $-3,1$ & 2,6 & 1,5 & $-1,6$ & 0,1 & $-0,1$ & $-1,1$ & $-3,4$ \\
\hline P02760 & Protein AMBP & 1,0 & 1,6 & 0,8 & 2,4 & $-0,1$ & 0,0 & $-1,7$ & 2,9 & 3,0 & 1,8 & 2,5 & 0,6 & 0,3 & $-1,4$ \\
\hline P02763 & Alpha-1-acid glycoprotein 1 & 0,1 & 3,2 & $-6,9$ & 1,7 & 1,2 & 3,9 & 1,6 & 2,0 & 4,6 & $-7,4$ & 2,1 & 1,9 & 4,2 & 1,8 \\
\hline P02769 & Serum Albumin & 0,4 & $-0,7$ & $-0,7$ & 1,7 & $-0,7$ & $-0,1$ & 0,4 & 2,3 & 0,7 & $-0,4$ & $-1,4$ & $-0,1$ & 0,3 & 0,6 \\
\hline P04004 & Vitronectin & $-1,8$ & $-4,0$ & $-1,3$ & $-0,2$ & $-1,0$ & $-2,9$ & $-2,9$ & 0,0 & $-4,1$ & $-0,3$ & 0,0 & $-0,4$ & $-3,0$ & $-4,3$ \\
\hline P04206 & Ig kappa chain V-III region GOL & $-3,9$ & $-1,0$ & $-0,3$ & 2,9 & $-1,1$ & $-5,7$ & $-2,6$ & $-2,3$ & 0,4 & 0,7 & 3,2 & $-0,5$ & $-6,9$ & $-2,9$ \\
\hline P04208 & Ig lambda chain V-I region WAH & 0,3 & $-3,0$ & 1,5 & 2,2 & 0,5 & $-1,8$ & $-1,9$ & 2,2 & $-3,1$ & 2,6 & 2,6 & 1,2 & $-2,0$ & $-3,3$ \\
\hline P04746 & Pancreatic alpha-amylase & 0,3 & $-0,9$ & $-0,9$ & $-2,9$ & $-0,6$ & 1,2 & $-0,5$ & $-2,3$ & 0,6 & 0,2 & $-2,8$ & 0,0 & 1,6 & $-0,4$ \\
\hline P05451 & Lithostathine-1-alpha & $-1,2$ & $-1,8$ & 0,6 & $-3,1$ & $-0,4$ & $-2,2$ & $-2,3$ & 0,6 & $-0,9$ & 1,7 & $-4,3$ & 0,2 & $-2,4$ & $-3,7$ \\
\hline P06702 & Protein $5100-\mathrm{A} 9$ & $-0,1$ & 0,0 & $-4,3$ & $-2,0$ & $-1,4$ & 0,0 & 0,3 & 1,7 & 1,2 & $-4,8$ & $-3,2$ & $-1,0$ & 0,2 & 0,0 \\
\hline P07858 & Cathepsin B & $-0,2$ & 2,8 & $-3,5$ & 1,1 & $-0,2$ & 0,7 & 0,3 & 1,7 & 4,3 & $-3,0$ & 1,4 & 0,5 & 1,0 & 0,3 \\
\hline P07911-5 & Isoform 5 of Uromodulin & $-2,0$ & $-2,6$ & $-1,0$ & $-2,1$ & $-0,6$ & $-1,4$ & $-0,7$ & 0,0 & $-1,1$ & 0,2 & $-1,6$ & 0,1 & $-1,0$ & $-0,5$ \\
\hline P07998 & Ribonuclease pancreatic & 2,8 & 3,4 & $-1,9$ & 0,7 & $-2,2$ & 1,8 & $-4,6$ & 4,7 & 4,8 & $-0,9$ & 1,0 & $-1,5$ & 2,2 & $-6,0$ \\
\hline P08571 & Monocyte differentiation antigen CD14 & $-6,5$ & $-3,9$ & $-1,5$ & 0,3 & 0,4 & $-5,9$ & $-0,7$ & $-6,1$ & $-3,0$ & $-0,4$ & 0,7 & 1,0 & $-7,2$ & $-0,6$ \\
\hline P09603 & Macrophage colony-stimulating factor 1 & $-1,6$ & $-0,4$ & $-4,5$ & $-0,6$ & $-1,9$ & 0,2 & 0,7 & 0,2 & 1,0 & $-4,0$ & $-0,4$ & $-1,3$ & 0,6 & 0,8 \\
\hline P10153 & Non-secretory ribonuclease & $-0,2$ & 2,1 & $-1,1$ & $-0,8$ & $-0,6$ & 1,2 & $-0,5$ & 1,7 & 3,4 & 0,0 & $-0,4$ & 0,1 & 1,5 & $-0,2$ \\
\hline P10253 & lysosomal alpha-glucosidase & 2,1 & $-1,0$ & $-0,4$ & $-2,6$ & $-0,4$ & 0,5 & $-1,2$ & $-4,0$ & 0,4 & 0,7 & $-2,5$ & 0,3 & 0,8 & $-1,1$ \\
\hline P12036 & Neurofilament heavy polypeptide & $-2,9$ & $-1,2$ & 0,1 & $-3,2$ & $-0,5$ & $-2,4$ & $-2,4$ & $-1,5$ & 0,0 & 1,1 & $-4,4$ & 0,2 & $-2,5$ & $-3,8$ \\
\hline P12830 & Cadherin-1 & 1,6 & 1,8 & $-2,3$ & 1,2 & 0,5 & 2,7 & 0,4 & 3,5 & 3,3 & $-1,3$ & 1,6 & 1,2 & 3,0 & 0,6 \\
\hline P13646 & Keratin, type I cytoskeletal 13 & $-1,3$ & $-2,4$ & $-0,1$ & $-2,6$ & $-0,7$ & $-1,8$ & $-3,1$ & 0,7 & $-0,9$ & 1,0 & $-2,3$ & 0,0 & $-1,5$ & $-3,1$ \\
\hline P13987 & CD59 glycoprotein & $-2,7$ & $-0,9$ & $-7,1$ & $-4,8$ & $-4,2$ & $-2,4$ & $-2,5$ & $-0,8$ & 0,5 & $-7,7$ & $-6,1$ & $-3,8$ & $-2,2$ & $-2,8$ \\
\hline P14384 & Carboxypeptidase M & $-3,0$ & $-2,0$ & $-3,3$ & $-3,3$ & 0,2 & 1,1 & $-2,5$ & $-1,6$ & $-1,1$ & $-2,5$ & $-4,5$ & 0,9 & 1,5 & $-3,9$ \\
\hline P14923 & Junction plakoglobin & $-2,5$ & 2,3 & $-0,4$ & 1,1 & 1,5 & 1,9 & $-0,5$ & $-2,1$ & 3,8 & 0,6 & 1,2 & 2,2 & 2,3 & $-1,8$ \\
\hline P15289-1 & arylsulfatase A & 3,0 & 2,0 & $-1,1$ & $-3,5$ & 0,3 & 2,5 & $-2,8$ & 4,9 & 3,5 & 0,0 & $-4,8$ & 1,0 & 2,9 & $-4,2$ \\
\hline P15924-1 & Desmoplakin & $-0,9$ & 0,9 & $-0,4$ & 0,4 & 1,4 & 1,3 & 0,6 & 0,8 & 2,3 & 0,7 & 0,5 & 2,1 & 1,7 & 0,6 \\
\hline P16070 & CD44 antigen & 0,3 & 0,9 & $-1,0$ & $-0,9$ & $-0,3$ & 0,9 & 0,7 & 2,2 & 2,4 & 0,1 & $-0,6$ & 0,3 & 1,3 & 1,0 \\
\hline P16278 & Beta-galactosidase & 2,7 & 0,2 & $-0,4$ & $-1,8$ & 1,0 & 0,9 & $-1,1$ & 4,7 & 1,4 & 0,6 & $-3,0$ & 1,7 & 1,2 & $-2,4$ \\
\hline P17900 & Ganglioside GM2 activator & $-4,1$ & $-0,3$ & $-2,3$ & $-0,5$ & $-2,7$ & $-2,0$ & $-2,1$ & $-3,8$ & 0,9 & $-1,4$ & $-0,4$ & $-2,4$ & $-2,2$ & $-3,4$ \\
\hline P19652 & Alpha-1-acid glycoprotein 2 & $-6,0$ & 0,0 & $-7,0$ & 1,9 & 0,1 & 0,3 & 0,6 & $-5,7$ & 1,4 & $-7,6$ & 2,2 & 0,8 & 0,6 & 0,8 \\
\hline P19961 & Alpha-amylase 2B & 0,4 & $-0,7$ & $-0,7$ & $-2,7$ & $-0,4$ & 1,4 & $-0,3$ & $-2,3$ & 0,7 & 0,3 & $-2,6$ & 0,2 & 1,7 & $-0,2$ \\
\hline P22105-4 & Isoform 5 of Tenascin-X & 0,5 & 1,5 & $-1,7$ & $-3,1$ & 0,6 & $-1,1$ & $-2,4$ & 2,4 & 2,9 & $-0,8$ & $-4,4$ & 1,3 & $-0,9$ & $-3,8$ \\
\hline P24821 & Tenascin & 3,7 & 3,0 & $-0,9$ & 1,4 & 1,5 & 1,7 & $-0,7$ & 5,7 & 4,5 & 0,0 & 1,6 & 2,2 & 2,1 & $-2,0$ \\
\hline P25311 & Zinc-alpha-2-glycoprotein & 1,9 & $-4,4$ & $-0,3$ & $-2,5$ & 0,6 & $-3,3$ & $-3,4$ & 3,9 & $-4,6$ & 0,8 & $-2,7$ & 1,3 & $-3,5$ & $-4,7$ \\
\hline P29508 & Serpin B3 & $-2,4$ & $-1,1$ & $-4,7$ & $-4,0$ & $-1,7$ & 1,9 & $-3,2$ & $-0,7$ & 0,2 & $-4,2$ & $-5,2$ & $-1,1$ & 2,3 & $-4,6$ \\
\hline P31944 & Caspase-14 & 1,1 & 1,3 & $-2,3$ & 1,6 & 2,2 & 0,8 & 2,4 & 2,9 & 2,4 & $-2,6$ & 1,6 & 3,0 & 0,9 & 2,3 \\
\hline P41222 & Prostaglandin- $\mathrm{H} 2 \mathrm{D}$-isomerase & 1,9 & 1,4 & 1,1 & 0,0 & 0,2 & 0,6 & $-0,5$ & 3,8 & 2,9 & 2,2 & 0,4 & 0,9 & 1,0 & $-0,3$ \\
\hline P43652 & Afamin & 3,3 & 3,5 & $-3,2$ & 3,5 & 0,4 & $-1,6$ & 2,2 & 5,3 & 5,0 & $-3,6$ & 3,9 & 1,0 & $-2,8$ & 2,3 \\
\hline P48594 & Serpin B4 & $-0,5$ & 0,2 & $-3,9$ & $-0,8$ & 0,1 & 2,7 & $-2,4$ & 1,3 & 1,6 & $-3,3$ & $-0,8$ & 0,7 & 3,1 & $-3,8$ \\
\hline P62736 & Actin, aortic smooth muscle & 0,7 & 0,7 & $-3,0$ & $-0,7$ & $-0,1$ & 1,2 & $-2,7$ & 2,6 & 2,1 & $-2,1$ & $-0,5$ & 0,6 & 1,5 & $-4,1$ \\
\hline P78324-2 & Isoform 2 of Tyrosine-protein phosphatase non-receptor type substrate 1 & $-2,5$ & $-3,1$ & $-0,3$ & $-0,5$ & 1,3 & $-2,0$ & 1,2 & $-1,1$ & $-3,2$ & 0,7 & $-0,4$ & 2,0 & $-2,1$ & 1,3 \\
\hline P81605-2 & Isoform 2 of Dermcidin & 1,2 & 2,1 & 1,2 & 2,5 & 2,7 & 3,6 & 2,7 & 3,2 & 3,5 & 2,3 & 2,9 & 3,4 & 4,0 & 2,9 \\
\hline P98160 & Basement membrane-specific heparan sulfate proteoglycan core protein & 1,5 & $-0,5$ & $-1,0$ & $-0,8$ & $-0,6$ & $-1,6$ & $-2,1$ & $-3,4$ & 0,9 & 0,1 & $-0,4$ & 0,1 & $-1,3$ & $-1,9$ \\
\hline P98164 & Low-density lipoprotein receptor-related protein 2 & $-3,2$ & 1,6 & $-3,0$ & 0,2 & 2,2 & $-1,5$ & 0,0 & $-1,9$ & 3,1 & $-2,2$ & 0,4 & $-2,9$ & $-1,3$ & 0,1 \\
\hline Q02413 & Desmoglein -1 & $-2,5$ & 3,0 & $-2,0$ & 1,6 & 1,8 & 2,4 & 2,7 & $-2,1$ & 4,4 & $-1,4$ & 1,8 & 2,5 & 2,8 & 2,8 \\
\hline Q07507 & Dermatopontin & $-4,8$ & $-2,3$ & 1,1 & $-0,1$ & $-0,6$ & $-4,3$ & $-1,2$ & $-4,5$ & $-1,4$ & 2,2 & 0,2 & 0,0 & $-5,5$ & $-1,5$ \\
\hline Q13228-4 & Isoform 4 of Selenium-binding protein 1 & 3,0 & $-1,6$ & $-2,0$ & 0,3 & $-0,7$ & $-0,4$ & $-0,5$ & 5,0 & $-1,7$ & $-1,4$ & 0,2 & $-0,1$ & $-0,5$ & $-1,8$ \\
\hline Q14624-1 & Inter-alpha-trypsin inhibitor heavy chain $\mathrm{H} 4$ & $-5,5$ & $-4,6$ & $-3,1$ & 0,7 & $-0,3$ & $-3,4$ & $-0,7$ & $-5,2$ & $-4,7$ & $-2,1$ & 1,0 & 0,4 & $-3,6$ & $-0,6$ \\
\hline Q16270-1 & Insulin-like growth factor-binding protein 7 & 0,3 & 0,7 & $-2,7$ & $-0,7$ & $-1,2$ & $-1,4$ & $-2,6$ & 2,3 & 2,2 & $-1,7$ & $-0,4$ & $-0,5$ & $-1,1$ & $-2,9$ \\
\hline Q5ZPR3 & CD276 antigen & $-4,1$ & 0,9 & $-3,6$ & $-2,8$ & 0,9 & $-2,0$ & 0,2 & $-3,8$ & 2,3 & $-3,0$ & $-4,1$ & 1,6 & $-2,2$ & 0,2 \\
\hline Q6EMK4 & vasorin & $-2,5$ & $-5,3$ & $-5,7$ & $-0,9$ & 1,1 & $-5,7$ & 0,2 & $-0,7$ & $-5,4$ & $-5,2$ & $-0,6$ & 1,8 & $-7,0$ & 0,4 \\
\hline Q6GTX8 & Leukocyte-associated immunoglobulin-like receptor 1 & 0,9 & $-0,1$ & $-0,2$ & $-2,5$ & $-0,2$ & $-2,3$ & $-2,9$ & 2,8 & 1,4 & 0,9 & $-2,3$ & 0,4 & $-2,0$ & $-3,2$ \\
\hline Q6ISS4 & Leukocyte-associated immunoglobulin-like receptor 2 & 0,6 & $-1,3$ & 0,3 & $-2,6$ & 0,0 & $-3,4$ & $-3,4$ & 2,6 & 0,0 & 1,3 & $-2,8$ & 0,7 & $-3,5$ & $-4,8$ \\
\hline Q8N1N4 & Keratin, type II cytoskeletal 78 & $-2,2$ & $-0,4$ & 0,3 & $-0,9$ & 0,0 & $-3,2$ & $-1,7$ & $-0,8$ & 0,7 & 1,4 & $-1,0$ & 0,6 & $-4,4$ & $-3,0$ \\
\hline Q8TDQ0 & Hepatitis A virus cellular receptor 2 & $-1,6$ & 1,3 & $-3,3$ & $-1,5$ & $-0,1$ & 0,0 & $-1,5$ & 0,2 & 2,8 & $-2,4$ & $-1,4$ & 0,6 & 0,3 & $-1,8$ \\
\hline Q8WVN6 & Secreted and transmembrane protein 1 & $-0,6$ & $-1,9$ & $-4,2$ & $-0,2$ & $-1,9$ & $-3,0$ & $-0,8$ & 1,3 & $-0,5$ & $-3,2$ & 0,2 & $-1,3$ & $-2,8$ & $-0,7$ \\
\hline Q969P0 & Immunoglobulin superfamily member 8 & $-3,3$ & $-2,3$ & 1,3 & $-0,8$ & $-0,3$ & $-2,1$ & $-1,3$ & $-1,9$ & $-1,4$ & 2,4 & $-0,6$ & 0,4 & $-2,0$ & $-1,6$ \\
\hline Q96FE7-1 & Phosphoinositide-3-kinase-interacting protein 1 & 0,8 & 0,3 & $-0,3$ & $-2,8$ & 0,1 & $-0,8$ & $-1,2$ & $-2,7$ & 1,8 & 0,8 & $-2,7$ & 0,8 & $-0,5$ & $-1,1$ \\
\hline Q96RW7-1 & Hemicentin-1 & 1,7 & 2,4 & $-0,6$ & $-0,8$ & 0,9 & 0,2 & $-2,3$ & 3,6 & 3,9 & 0,4 & $-0,7$ & 1,5 & 0,6 & $-3,7$ \\
\hline Q99715-1 & Collagen alpha- 1 (XII) chain & 1,3 & 1,6 & $-2,5$ & 1,2 & $-0,3$ & $-1,0$ & $-2,3$ & $-3,3$ & 3,0 & $-1,7$ & 1,5 & 0,3 & $-0,8$ & $-3,7$ \\
\hline Q9H299 & SH3 domain-binding glutamic acid-rich-like protein 3 & 2,0 & 2,5 & 4,1 & $-1,1$ & 0,2 & $-0,3$ & $-0,3$ & 4,0 & 4,0 & 5,2 & $-2,2$ & 0,8 & $-0,4$ & $-1,6$ \\
\hline Q9H3G5 & Probable serine carboxypeptidase CPVL & 0,3 & 2,9 & $-0,7$ & 0,0 & 0,6 & 0,8 & 0,8 & 1,9 & 4,4 & 0,0 & $-1,0$ & 1,3 & 0,9 & $-0,4$ \\
\hline Q9HCUO & endosialin & $-1,8$ & $-0,3$ & 0,5 & $-3,4$ & 0,5 & $-4,9$ & $-3,4$ & 0,1 & 1,2 & 1,6 & $-3,3$ & 1,2 & $-5,1$ & $-3,7$ \\
\hline Q9ULI3 & Protein HEG homolog 1 & $-1,6$ & $-1,4$ & $-5,0$ & $-0,3$ & $-0,1$ & $-1,1$ & $-1,9$ & 0,0 & $-0,5$ & $-5,5$ & $-0,3$ & 0,5 & $-1,0$ & $-3,3$ \\
\hline Q9UNN8 & Endothelial protein $\mathrm{C}$ receptor & $-5,3$ & $-5,9$ & $-1,3$ & $-4,0$ & $-0,1$ & $-4,8$ & $-2,5$ & $-3,9$ & $-6,1$ & $-0,3$ & $-4,2$ & 0,6 & $-5,0$ & $-2,6$ \\
\hline
\end{tabular}




\section{Kidney Blood Pressure Research}

Caterino et al.: Urine Proteome of Patients with Bardet-Biedl Syndrome

Table 4. Urine protein abundance (fold changes) based on $\mathrm{R}_{\mathrm{SC}}$ and $\mathrm{R}_{\mathrm{NSAF}}$ in male BBS subjects

\begin{tabular}{|c|c|c|c|c|c|c|c|c|c|c|c|c|c|c|c|}
\hline \multirow[b]{2}{*}{ Accession } & \multirow[b]{2}{*}{ Description } & \multicolumn{7}{|c|}{$\mathrm{R}_{\mathrm{sc}}$} & \multicolumn{7}{|c|}{$\mathrm{R}_{\mathrm{NSAF}}$} \\
\hline & & $\mathrm{Y} 1$ & Y2 & Y3 & Y4 & Y5 & Y6 & Y7 & Y1 & Y2 & Y3 & $\mathrm{Y} 4$ & Y5 & Y6 & Y7 \\
\hline B9A064-1 & Immunoglobulin lambda-like polypeptide 5 & $-2,7$ & $-2,8$ & $-1,0$ & $-1,8$ & $-0,9$ & 0,7 & $-1,4$ & $-4,5$ & $-4,6$ & $-1,4$ & $-1,7$ & $-1,7$ & 0,5 & $-1,3$ \\
\hline 075594 & peptidoglycan recognition protein 1 & 1,0 & $-2,1$ & $-4,2$ & $-3,7$ & $-1,4$ & $-3,4$ & 0,0 & 0,8 & $-2,6$ & $-5,7$ & $-3,8$ & $-2,1$ & $-4,2$ & 0,2 \\
\hline P00734 & Prothrombin & 0,2 & $-0,7$ & 0,3 & 0,6 & 0,4 & 1,2 & $-0,6$ & $-0,1$ & $-1,0$ & 0,4 & 0,7 & $-0,3$ & 1,0 & $-0,4$ \\
\hline P01011-1 & Alpha-1-antichymotrypsin & $-3,4$ & $-1,1$ & $-3,2$ & $-1,6$ & $-1,9$ & $-0,6$ & $-2,1$ & $-5,2$ & $-1,6$ & $-4,7$ & $-1,6$ & $-2,7$ & $-0,8$ & $-2,0$ \\
\hline P01042-2 & Isoform LMW of Kininogen-1 & $-2,3$ & 0,9 & $-3,5$ & 0,2 & $-5,2$ & 0,0 & $-3,0$ & $-2,8$ & 0,7 & $-3,9$ & 0,3 & $-6,5$ & $-0,2$ & $-2,8$ \\
\hline P01620 & Ig kappa chain V-III region SIE & 3,3 & 0,2 & $-0,3$ & $-1,0$ & 5,2 & $-2,7$ & 0,4 & 3,2 & $-0,1$ & $-0,6$ & $-0,9$ & 4,6 & $-4,3$ & 0,7 \\
\hline P01622 & Ig kappa chain V-III region TI & 3,7 & 0,6 & 0,1 & $-1,0$ & 5,6 & $-2,3$ & 0,3 & 3,6 & 0,3 & $-0,2$ & $-0,9$ & 5,0 & $-3,9$ & 0,6 \\
\hline P01623 & Ig kappa chain V-III region WOL & 3,3 & 0,2 & $-0,3$ & $-0,7$ & 5,2 & $-2,7$ & 0,4 & 3,2 & $-0,1$ & $-0,6$ & $-0,6$ & 4,6 & $-4,3$ & 0,7 \\
\hline P01833 & Polymeric immunoglobulin receptor & $-4,1$ & $-4,2$ & 0,2 & $-0,6$ & $-1,0$ & 0,1 & $-1,4$ & $-6,0$ & $-6,0$ & 0,3 & $-0,5$ & $-1,8$ & $-0,1$ & $-1,2$ \\
\hline P01834 & Ig kappa chain $\mathrm{C}$ region & $-3,1$ & $-1,4$ & $-1,4$ & $-0,2$ & 1,1 & 0,8 & $-3,0$ & $-3,7$ & $-1,7$ & $-1,4$ & $-0,1$ & 0,4 & 0,6 & $-2,9$ \\
\hline P01876 & Ig alpha- 1 chain $\mathrm{C}$ region & 1,2 & 1,9 & $-3,6$ & $-1,7$ & $-4,6$ & $-5,9$ & $-0,4$ & 1,0 & 1,6 & $-4,1$ & $-1,6$ & $-5,7$ & $-7,8$ & $-0,2$ \\
\hline P02749 & Beta-2-glycoprotein 1 & $-4,1$ & $-4,2$ & 0,2 & $-0,4$ & 0,1 & 1,3 & $-1,2$ & $-6,0$ & $-6,0$ & 0,3 & $-0,3$ & $-0,6$ & 1,1 & $-1,0$ \\
\hline P02750 & Leucine-rich alpha-2-glycoprotein & $-0,3$ & $-1,2$ & $-2,3$ & $-3,8$ & $-3,3$ & $-4,6$ & $-3,2$ & $-0,6$ & $-1,6$ & $-2,7$ & $-4,0$ & $-4,3$ & $-6,4$ & $-3,2$ \\
\hline P02751 & Fibronectin & 4,2 & 1,1 & 0,1 & 1,7 & $-0,3$ & $-1,4$ & $-0,3$ & 4,0 & 0,9 & 0,1 & 1,9 & $-1,0$ & $-2,0$ & $-0,1$ \\
\hline P02760 & Protein AMBP & 0,2 & 0,9 & 0,2 & 0,1 & 1,1 & 1,5 & $-0,6$ & $-0,1$ & 0,6 & 0,3 & 0,1 & 0,2 & 1,1 & $-0,3$ \\
\hline P02769 & Serum Albumin & 0,6 & 3,2 & $-0,6$ & 1,2 & $-2,3$ & 1,3 & $-5,0$ & 0,3 & 3,0 & $-0,6$ & 1,4 & $-3,2$ & 1,1 & $-6,3$ \\
\hline P04004 & Vitronectin & 0,4 & $-1,2$ & $-1,0$ & 2,1 & 3,2 & 2,4 & $-1,1$ & $-0,1$ & $-2,8$ & $-2,3$ & 2,5 & 2,7 & 2,5 & $-1,1$ \\
\hline P04206 & Ig kappa chain V-III region GOL & 3,7 & 0,6 & 0,8 & $-1,0$ & 5,6 & $-2,3$ & 0,3 & 3,6 & 0,3 & 0,8 & $-0,9$ & 5,1 & $-3,9$ & 0,6 \\
\hline P04208 & Ig lambda chain V-I region WAH & 3,0 & 5,3 & 1,3 & $-2,6$ & 1,1 & 1,2 & $-0,3$ & 3,2 & 5,6 & 1,4 & $-3,6$ & 0,8 & 1,3 & $-0,1$ \\
\hline P04433 & Ig kappa chain V-III region VG & $-3,4$ & $-1,9$ & $-0,4$ & $-5,5$ & $-1,6$ & $-2,4$ & $-2,5$ & $-5,2$ & $-2,6$ & $-0,5$ & $-7,0$ & $-2,4$ & $-3,2$ & $-2,6$ \\
\hline P04746 & Pancreatic alpha-amylase & $-3,1$ & 0,4 & $-0,4$ & $-1,5$ & $-2,9$ & $-5,0$ & $-0,3$ & $-3,5$ & 0,2 & $-0,3$ & $-1,4$ & $-3,7$ & $-5,7$ & $-0,1$ \\
\hline P05154 & Plasma serine protease inhibitor & $-1,1$ & $-1,4$ & $-0,8$ & $-2,0$ & $-3,2$ & $-1,7$ & $-1,0$ & $-1,4$ & $-1,7$ & $-0,8$ & $-1,9$ & $-4,0$ & $-2,0$ & $-0,8$ \\
\hline P07858 & Cathepsin B & $-0,4$ & 1,1 & $-0,3$ & 2,0 & $-0,5$ & 1,2 & $-0,3$ & $-1,7$ & 0,9 & $-1,3$ & 2,7 & $-1,2$ & 1,3 & $-0,1$ \\
\hline P07911-5 & Isoform 5 of Uromodulin & 0,3 & 0,4 & 2,4 & $-0,2$ & $-0,8$ & 0,2 & 0,5 & 0,1 & 0,1 & 2,4 & $-0,1$ & $-1,6$ & 0,0 & 0,7 \\
\hline P07998 & Ribonuclease pancreatic & 1,4 & 0,3 & 0,7 & 1,2 & 0,8 & 1,6 & 0,6 & 1,1 & 0,1 & 0,8 & 1,3 & 0,1 & 1,3 & 0,8 \\
\hline P08571 & Monocyte differentiation antigen CD14 & 1,6 & $-1,2$ & $-1,6$ & $-0,1$ & $-2,8$ & $-3,6$ & $-0,6$ & 1,4 & $-1,5$ & $-1,7$ & 0,0 & $-3,6$ & $-4,4$ & $-0,4$ \\
\hline P09564 & T-cell antigen $\mathrm{CD} 7$ & 4,9 & 2,7 & 2,9 & 2,4 & 2,7 & 0,5 & 2,5 & 6,2 & 3,5 & 4,0 & 4,0 & 3,4 & 0,3 & 4,1 \\
\hline P09603 & Macrophage colony-stimulating factor 1 & 3,5 & 1,1 & 5,7 & 4,9 & 1,8 & 2,8 & 2,5 & 4,6 & 0,9 & 7,3 & 6,6 & 2,4 & 3,9 & 4,1 \\
\hline P0CG05 & Ig lambda- 2 chain $\mathrm{C}$ regions & $-3,1$ & $-0,4$ & $-1,4$ & $-2,1$ & $-0,8$ & 1,1 & $-1,8$ & $-4,9$ & $-0,7$ & $-1,8$ & $-2,1$ & $-1,6$ & 0,9 & $-1,7$ \\
\hline P0DJD7 & Pepsin A-4 & 1,2 & $-1,7$ & $-4,6$ & $-2,2$ & $-3,3$ & 1,4 & 1,1 & 0,9 & $-3,4$ & 4,9 & $-2,5$ & $-5,5$ & 1,3 & 1,5 \\
\hline P10153 & Non-secretory ribonuclease & $-0,1$ & $-0,5$ & $-1,9$ & 2,2 & 1,5 & 1,3 & 1,0 & $-0,5$ & $-0,8$ & $-2,3$ & 2,3 & 0,8 & 1,1 & 1,2 \\
\hline P10253 & lysosomal alpha-glucosidase & 0,8 & $-0,7$ & $-1,0$ & 0,4 & $-2,6$ & $-1,6$ & 0,1 & 0,6 & $-1,0$ & $-1,0$ & 0,5 & $-3,4$ & $-1,9$ & 0,4 \\
\hline P10451-5 & Isoform 5 of Osteopontin & $-2,1$ & 1,1 & $-2,3$ & $-1,0$ & $-4,7$ & $-0,3$ & $-1,5$ & $-2,5$ & 0,9 & $-2,3$ & $-0,9$ & $-5,6$ & $-0,6$ & $-1,2$ \\
\hline P10909-2 & Isoform 2 of Clusterin & 2,0 & 1,9 & $-0,9$ & $-2,4$ & $-3,4$ & $-4,0$ & $-1,2$ & 1,8 & 1,7 & $-1,1$ & $-2,4$ & $-4,7$ & $-5,8$ & $-1,0$ \\
\hline P12830 & Cadherin-1 & $-1,4$ & $-2,7$ & 2,0 & 1,3 & 0,4 & 1,4 & 0,5 & $-1,8$ & $-3,5$ & 2,1 & 1,4 & $-0,3$ & 1,2 & 0,7 \\
\hline P13646 & Keratin, type I cytoskeletal 13 & 0,0 & $-0,8$ & $-2,2$ & 0,4 & 0,4 & $-3,0$ & 1,2 & $-0,4$ & $-1,5$ & $-3,6$ & 0,6 & $-0,2$ & $-4,7$ & 1,5 \\
\hline P15289-1 & arylsulfatase $\mathrm{A}$ & 0,1 & $-0,4$ & 2,6 & 2,4 & $-0,1$ & $-3,7$ & 0,9 & $-0,2$ & $-0,7$ & 2,7 & 2,6 & $-0,8$ & $-5,5$ & 1,2 \\
\hline P15586 & $\mathrm{N}$-acetylglucosamine-6-sulfatase & $-4,4$ & $-2,2$ & $-2,7$ & $-2,9$ & $-1,4$ & $-5,1$ & $-2,7$ & $-6,2$ & $-2,7$ & $-3,1$ & $-2,9$ & $-2,2$ & $-6,9$ & $-2,6$ \\
\hline P16070 & CD44 antigen & 0,8 & 2,8 & 1,6 & 2,4 & $-0,9$ & 1,2 & 2,0 & 0,5 & 2,7 & 1,7 & 2,6 & $-1,7$ & 1,0 & 2,3 \\
\hline P18135 & Ig kappa chain V-III region НАH & 2,9 & $-0,1$ & $-2,2$ & $-1,3$ & 5,0 & $-3,0$ & $-0,1$ & 2,8 & $-0,5$ & $-3,6$ & $-1,2$ & 4,3 & $-4,7$ & 0,1 \\
\hline P22105-4 & Isoform 5 of Tenascin-X & $-1,3$ & $-3,7$ & $-3,5$ & 0,2 & $-2,9$ & $-0,5$ & $-0,2$ & $-1,8$ & $-5,5$ & $-5,0$ & 0,3 & $-3,9$ & $-0,8$ & 0,0 \\
\hline P24821 & Tenascin & 2,8 & 2,7 & 4,5 & 5,2 & 1,8 & 4,9 & 2,8 & 3,6 & 3,5 & 6,0 & 6,9 & 2,4 & 6,3 & 4,5 \\
\hline P25311 & Zinc-alpha-2-glycoprotein & $-5,3$ & $-1,0$ & $-2,0$ & $-1,2$ & $-2,0$ & $-4,4$ & $-2,0$ & $-7,1$ & $-1,3$ & $-2,1$ & $-1,1$ & $-2,8$ & $-5,1$ & $-1,8$ \\
\hline P30530 & Tyrosine-protein kinase receptor UFO & 0,3 & 4,5 & $-0,3$ & 1,2 & $-3,7$ & $-2,7$ & 1,2 & $-0,1$ & 4,4 & $-0,6$ & 1,4 & $-5,9$ & $-4,3$ & 1,6 \\
\hline P41222 & Prostaglandin-H2 D-isomerase & 1,6 & $-0,6$ & 1,0 & $-0,5$ & 1,0 & 1,7 & $-0,2$ & 1,3 & $-0,8$ & 1,0 & $-0,4$ & 0,3 & 1,5 & 0,0 \\
\hline P55290-4 & Isoform 4 of Cadherin-13 & $-0,1$ & 2,4 & 2,3 & $-0,2$ & $-4,6$ & $-2,0$ & 0,9 & $-0,5$ & 2,2 & 2,5 & 0,0 & $-6,9$ & $-2,7$ & 1,2 \\
\hline P78324-2 & Isoform 2 of Tyrosine-protein phosphatase non-receptor type substrate 1 & 1,9 & 1,8 & $-0,3$ & 0,9 & $-0,5$ & $-1,1$ & 0,9 & 1,9 & 1,9 & $-1,3$ & 1,4 & $-1,2$ & $-2,3$ & 1,5 \\
\hline P80748 & Ig lambda chain V-III region LOI & 0,9 & 0,8 & 0,1 & $-2,2$ & 0,8 & 0,7 & 0,3 & 0,6 & 0,5 & 0,1 & $-2,2$ & 0,1 & 0,6 & 0,6 \\
\hline P81605-2 & Isoform 2 of Dermcidin & $-2,5$ & $-2,6$ & $-0,8$ & $-3,1$ & $-1,0$ & $-0,9$ & 0,4 & $-4,3$ & $-4,4$ & $-1,2$ & $-3,5$ & $-1,8$ & $-1,3$ & 0,6 \\
\hline P98160 & Basement membrane-specific heparan sulfate proteoglycan core protein & 1,4 & $-0,4$ & $-1,6$ & $-0,4$ & $-1,8$ & $-0,6$ & $-0,4$ & 1,1 & $-0,6$ & $-1,5$ & $-0,3$ & $-2,5$ & $-0,8$ & $-0,1$ \\
\hline Q02413 & Desmoglein-1 & 0,0 & $-2,4$ & 2,1 & $-0,1$ & $-2,4$ & $-3,0$ & 1,0 & $-0,4$ & $-4,1$ & 2,2 & 0,1 & $-3,6$ & $-4,7$ & 1,3 \\
\hline Q08380 & Galectin-3-binding protein & 1,8 & $-1,2$ & $-1,0$ & 0,0 & $-0,4$ & $-0,6$ & 1,1 & 1,5 & $-1,6$ & $-1,0$ & 0,2 & $-1,2$ & $-0,9$ & 1,3 \\
\hline Q14624-1 & Inter-alpha-trypsin inhibitor heavy chain $\mathrm{H} 4$ & 2,7 & 2,7 & $-4,5$ & $-1,7$ & $-2,5$ & $-5,2$ & $-0,3$ & 2,4 & 2,4 & $-6,0$ & $-1,6$ & $-3,4$ & $-7,0$ & $-0,1$ \\
\hline Q15113 & Procollagen C-endopeptidase enhancer 1 & 1,2 & $-0,5$ & $-0,3$ & 0,6 & 2,8 & 0,5 & $-0,3$ & 0,9 & $-1,8$ & $-1,3$ & 1,1 & 2,6 & 0,3 & $-0,1$ \\
\hline Q16270-1 & Insulin-like growth factor-binding protein 7 & $-1,0$ & $-1,1$ & $-3,2$ & $-0,3$ & $-1,3$ & $-0,1$ & $-1,7$ & $-1,5$ & $-1,6$ & $-4,7$ & $-0,2$ & $-2,1$ & $-0,4$ & $-1,6$ \\
\hline Q6EMK4 & vasorin & 0,5 & 0,2 & $-3,5$ & $-1,5$ & $-4,8$ & $-3,2$ & $-1,1$ & 0,3 & $-0,1$ & $-3,7$ & $-1,4$ & $-5,7$ & $-3,5$ & $-0,9$ \\
\hline Q6GTX8 & Leukocyte-associated immunoglobulin-like receptor 1 & $-2,3$ & 1,3 & 0,4 & 0,1 & 0,7 & $-3,2$ & $-0,6$ & 2,1 & 1,1 & 0,4 & 0,3 & 0,0 & $-4,9$ & $-0,4$ \\
\hline Q7Z5L0-1 & Vitelline membrane outer layer protein 1 homolog & 1,0 & 2,1 & 0,5 & $-0,8$ & $-1,7$ & $-2,0$ & $-1,4$ & 0,8 & 1,9 & 0,6 & $-0,6$ & $-2,5$ & $-2,4$ & $-1,2$ \\
\hline Q8N1N4 & Keratin, type II cytoskeletal 78 & $-1,2$ & $-1,2$ & 1,3 & 1,7 & 1,3 & 1,0 & 1,0 & $-2,7$ & $-2,8$ & 1,4 & 2,1 & 0,8 & 0,9 & 1,5 \\
\hline Q8WVN6 & Secreted and transmembrane protein 1 & 4,8 & 0,3 & $-1,0$ & 0,1 & 2,5 & $-1,8$ & 0,8 & 4,9 & $-0,1$ & $-2,3$ & 0,4 & 2,0 & $-3,3$ & 1,2 \\
\hline Q8WZ75-1 & Roundabout homolog 4 & 0,2 & $-3,0$ & $-2,8$ & $-0,9$ & $-6,9$ & $-5,9$ & $-0,4$ & 0,0 & $-3,5$ & $-3,0$ & $-0,8$ & $-9,2$ & $-7,7$ & $-0,2$ \\
\hline Q96FE7-1 & Phosphoinositide-3-kinase-interacting protein 1 & 0,9 & 2,2 & 0,7 & $-0,8$ & $-2,3$ & 0,3 & $-0,8$ & 0,6 & 2,0 & 0,8 & $-0,7$ & $-3,1$ & 0,1 & $-0,6$ \\
\hline Q99715-1 & Collagen alpha-1(XII) chain & 1,2 & 1,1 & $-1,0$ & 3,4 & 0,3 & 2,9 & 1,4 & 0,9 & 0,9 & $-2,3$ & 3,8 & $-0,2$ & 3,0 & 1,9 \\
\hline Q9HAT2 & sialate 0 -acetylesterase & 0,9 & $-0,8$ & $-2,2$ & $-0,6$ & $-2,4$ & $-3,0$ & $-1,0$ & 0,6 & $-1,5$ & $-3,6$ & $-0,4$ & $-3,6$ & $-4,7$ & $-0,9$ \\
\hline Q9HCU0 & endosialin & 1,8 & 1,2 & 0,7 & 0,3 & $-1,2$ & $-1,2$ & 0,0 & 1,5 & 1,0 & 0,7 & 0,4 & $-2,0$ & $-1,5$ & 0,2 \\
\hline Q9ULI3 & Protein HEG homolog 1 & $-2,7$ & $-2,8$ & 0,2 & 1,6 & 0,5 & 1,0 & 0,8 & $-4,5$ & $-4,6$ & 0,2 & 1,8 & $-0,2$ & 0,8 & 1,1 \\
\hline Q9UNN8 & Endothelial protein $\mathrm{C}$ receptor & 3,0 & $-0,8$ & $-2,2$ & $-2,2$ & $-1,6$ & $-3,0$ & 1,0 & 2,8 & $-1,5$ & $-3,6$ & $-2,2$ & $-2,6$ & $-4,7$ & 1,3 \\
\hline
\end{tabular}



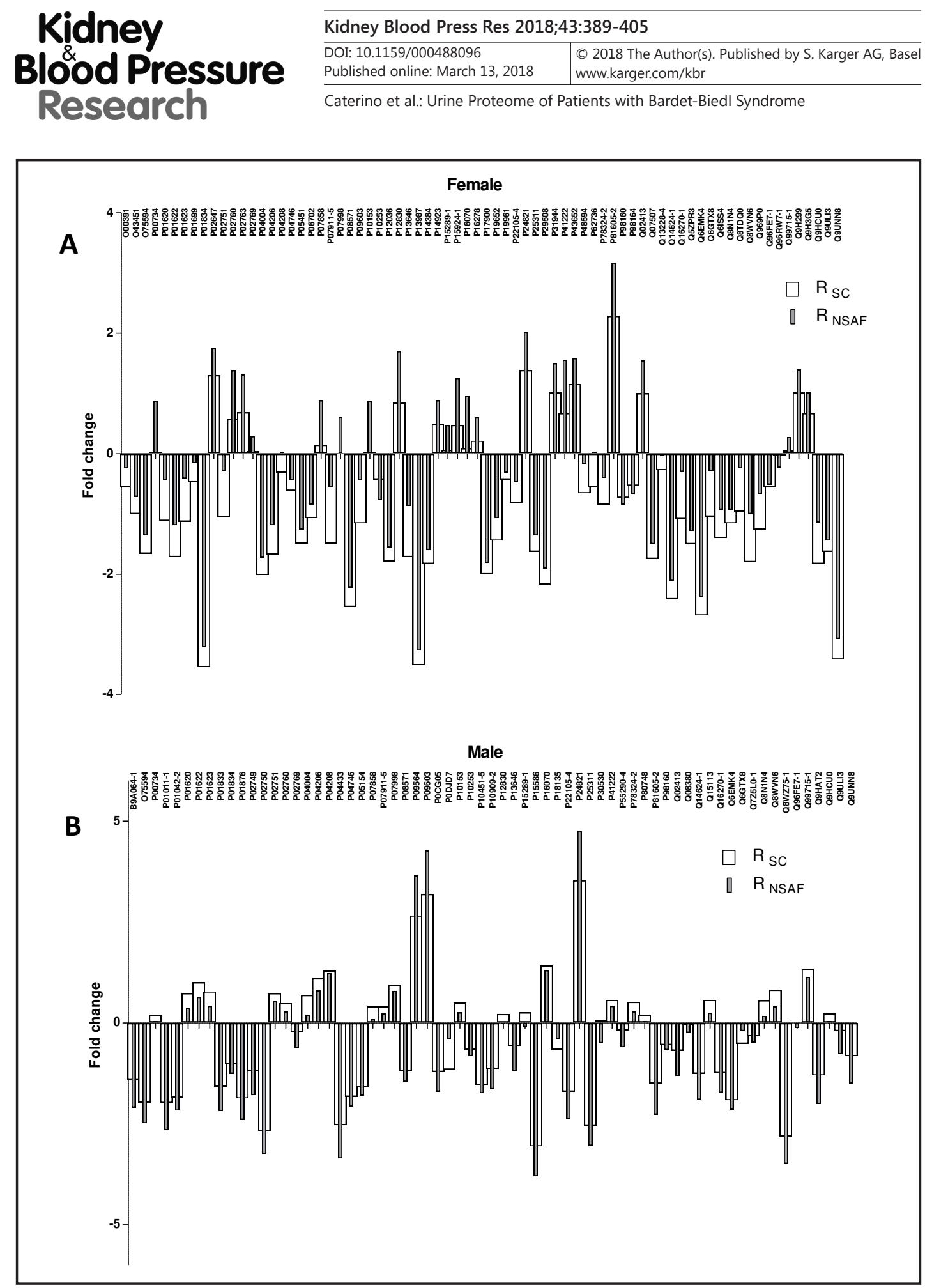

Fig. 2. Urine protein abundance (fold changes) based on Rsc and $\mathrm{R}_{\mathrm{NSAF}}$ parameters in female (A) and male patients (B) compared with the control. The Fig. shows that both methods used for protein quantization gives similar results, supporting the accuracy of the quantization. 


\section{Kidney \\ Blood Pressure Research}

Table 5. List of urine proteins deregulated in both female and male BBS subjects

\begin{tabular}{|c|c|}
\hline Accession & Description \\
\hline P15289-1 & Arylsulfatase A \\
\hline P98160 & Basement membrane-specific heparan sulfate proteoglycan core protein \\
\hline P12830 & Cadherin-1 \\
\hline P07858 & Cathepsin B \\
\hline P16070 & CD44 antigen \\
\hline Q99715-1 & Collagen alpha- 1 (XII) chain \\
\hline Q02413 & Desmoglein-1 \\
\hline Q9HCU0 & endosialin \\
\hline Q9UNN8 & Endothelial protein $\mathrm{C}$ receptor \\
\hline P01834 & Ig kappa chain $\mathrm{C}$ region \\
\hline P04206 & Ig kappa chain V-III region GOL \\
\hline P01620 & Ig kappa chain V-III region SIE \\
\hline P01622 & Ig kappa chain V-III region TI \\
\hline P01623 & Ig kappa chain V-III region WOL \\
\hline P04208 & Ig lambda chain V-I region WAH \\
\hline Q16270-1 & Insulin-like growth factor-binding protein 7 \\
\hline Q14624-1 & Inter-alpha-trypsin inhibitor heavy chain $\mathrm{H} 4$ \\
\hline P02751 & Fibronectin \\
\hline P81605-2 & Isoform 2 of Dermcidin \\
\hline P78324-2 & Isoform 2 of Tyrosine-protein phosphatase non-receptor type substrate 1 \\
\hline P22105-4 & Isoform 5 of Tenascin-X \\
\hline P07911-5 & Isoform 5 of Uromodulin \\
\hline P13646 & Keratin, type I cytoskeletal 13 \\
\hline Q8N1N4 & Keratin, type II cytoskeletal 78 \\
\hline Q6GTX8 & Leukocyte-associated immunoglobulin-like receptor 1 \\
\hline P10253 & lysosomal alpha-glucosidase \\
\hline P09603 & Macrophage colony-stimulating factor 1 \\
\hline P08571 & Monocyte differentiation antigen CD14 \\
\hline P10153 & Non-secretory ribonuclease \\
\hline P04746 & Pancreatic alpha-amylase \\
\hline 075594 & peptidoglycan recognition protein 1 \\
\hline Q96FE7-1 & Phosphoinositide-3-kinase-interacting protein 1 \\
\hline P41222 & Prostaglandin-H2 D-isomerase \\
\hline P02760 & Protein AMBP \\
\hline Q9ULI3 & Protein HEG homolog 1 \\
\hline P00734 & Prothrombin \\
\hline P07998 & Ribonuclease pancreatic \\
\hline Q8WVN6 & Secreted and transmembrane protein 1 \\
\hline P02769 & Serum Albumin \\
\hline P24821 & Tenascin \\
\hline Q6EMK4 & vasorin \\
\hline P04004 & Vitronectin \\
\hline P25311 & Zinc-alpha-2-glycoprotein \\
\hline
\end{tabular}

Fig. 3. Urine protein abundance (fold changes, based on $\mathrm{R}_{\mathrm{NSAF}}$ ) limited to proteins de-regulated in both female and male patients. The picture shows that most proteins are over or under-represented in both males and females, with only few proteins showing opposite behavior.

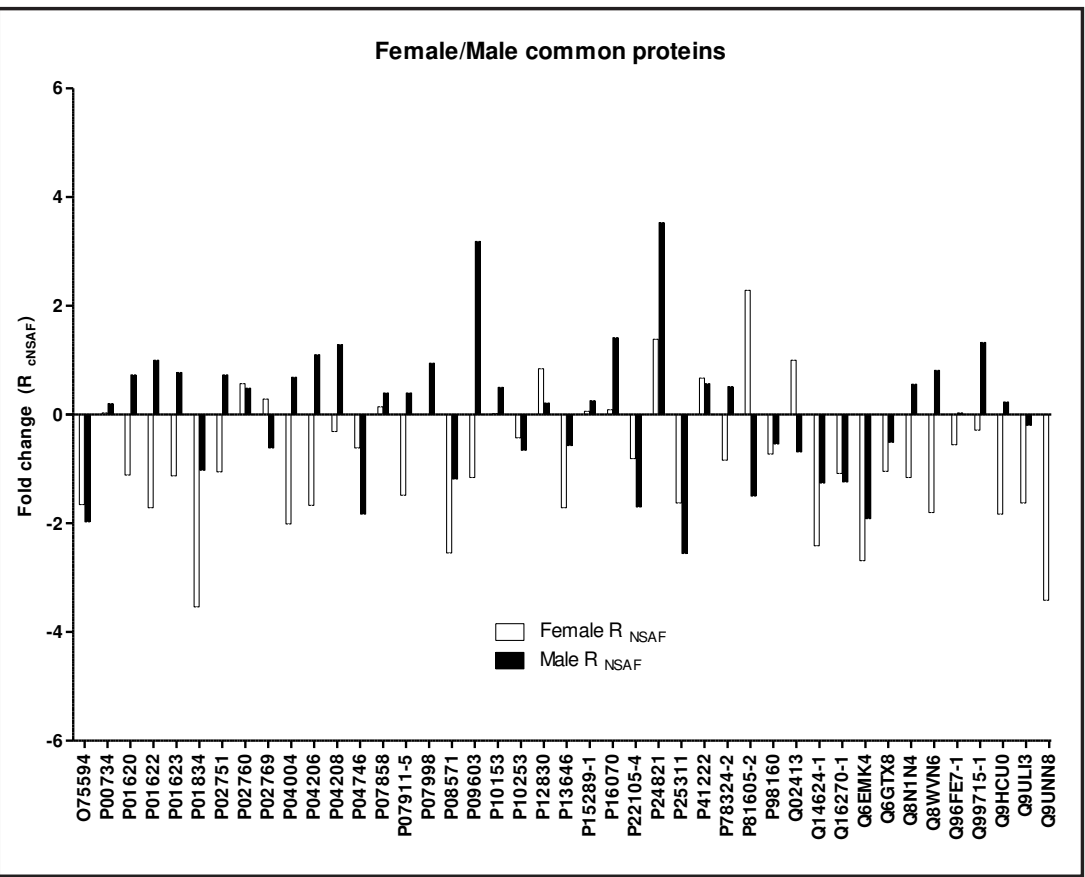




\section{Kidney Blood Pressure Research}

Fig. 4. Urinary BBS proteomes were clusterized by using STRING database. According to "Gene Ontology" (GO), the most significant biological processes were "Cell adhesion" and "Extracellular matrix organization". Proteins identified in BBS proteome involved in "Cell adhesion" and in "Extracellular matrix organization" biological processes are shown in red.

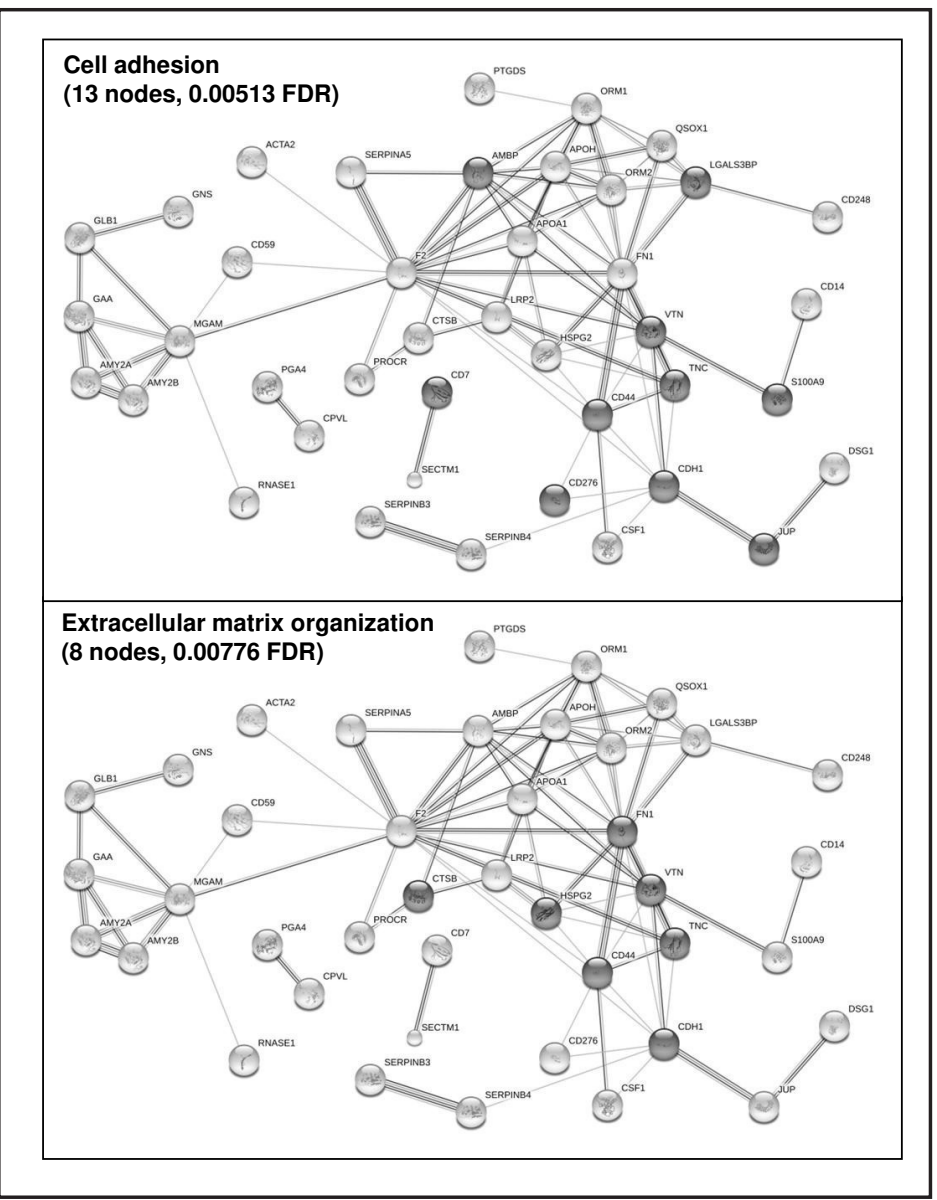

Fig. 5. Correlation between eGFR and urine Fibronectin, CD44 antigen and lysosomal alphaglucosidase in BBS patients $(n=14)$. eGFR is expressed in $\mathrm{ml} / \mathrm{min} / 1.73 \mathrm{~m}^{2}$.

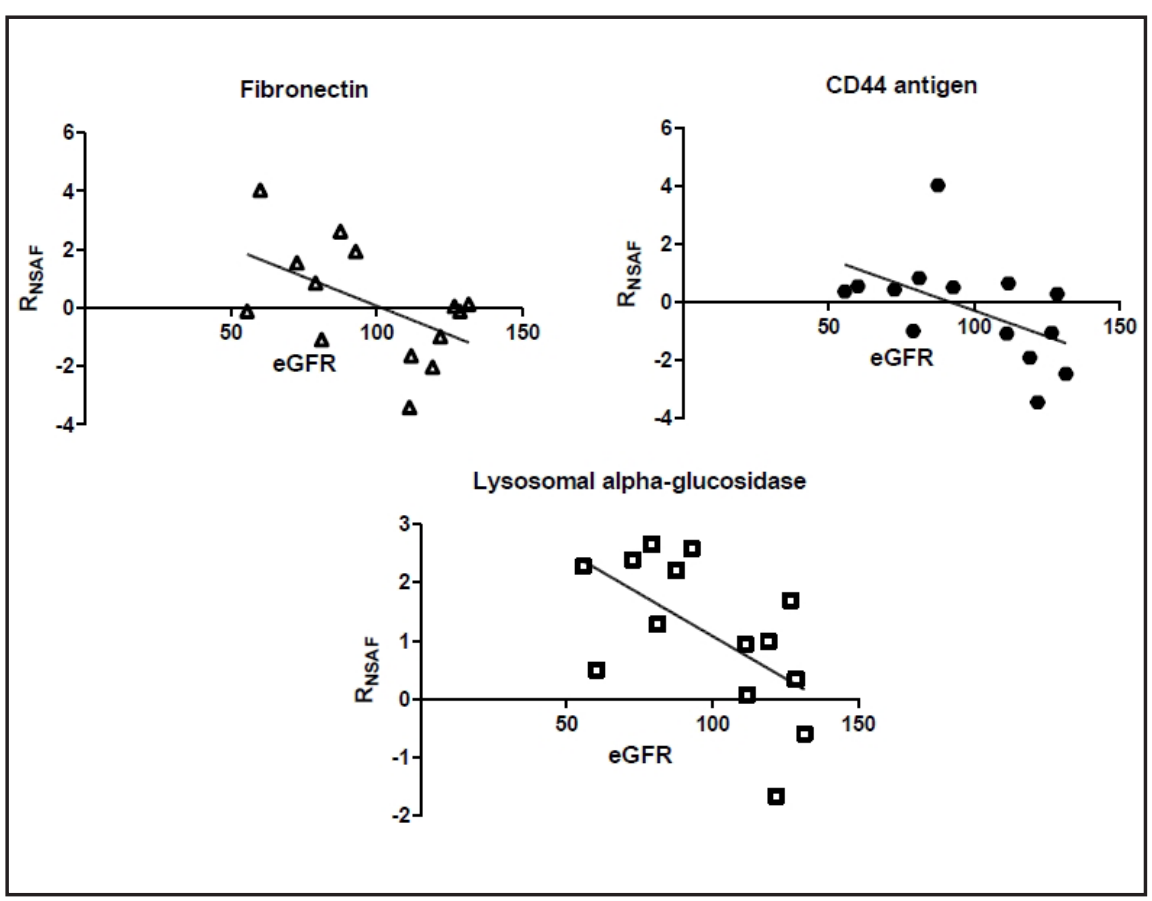




\section{Kidney \\ Blood Pressure Research}

Fig. 6. Correlation between annual $\triangle \mathrm{eGFR}$ and urine Fibronectin in BBS patients $(\mathrm{n}=$ 14). $\triangle \mathrm{eGFR}$ is expressed as $\mathrm{ml} / \mathrm{min} / 1.73 \mathrm{~m}^{2}$ per year. Statistical significance was determined by ANOVA.

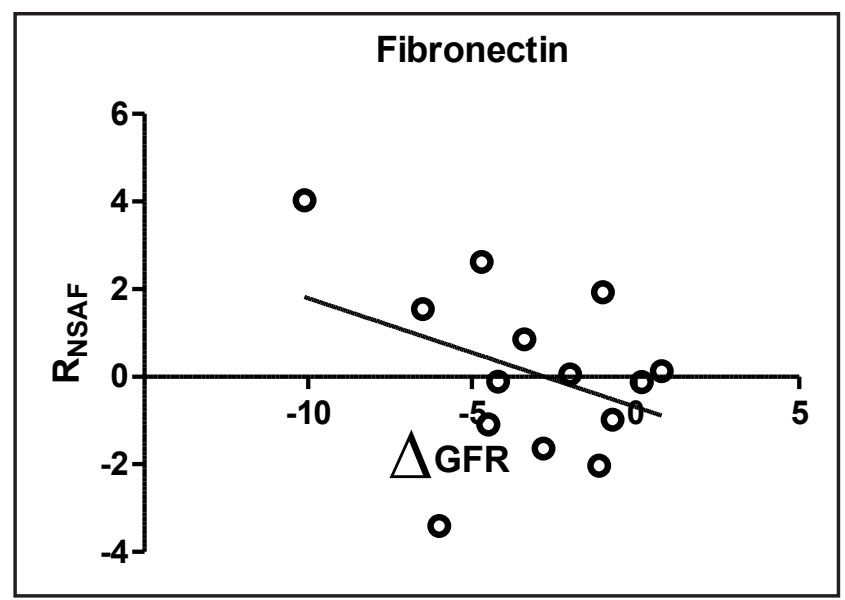

\section{Discussion}

This is the first study to our knowledge that analyzes urine proteome in a population of adult BBS patients and correlates the spectrum of urinary proteins with renal function.

There is no doubt that urine proteomics is an emerging field that has provided new insights in the understanding of the patho-physiological mechanisms of renal and extrarenal diseases [31]. Urine is a biological fluid that can be easily collected in a non-invasive manner, and it is an important source of renal and extra-renal proteins and peptides [19]; this has raised much attention on the potentiality of this tool in the discovery of disease biomarkers [32].

Given the large genetic heterogeneity of BBS, laborious genetic tests are required for a molecular screening of patients. However, to date the diagnosis is mainly based on clinical findings [5]. Proteomics screening may be potentially of help in (I) identifying diseasespecific biomarkers, useful for a more accurate diagnosis, and (II) in identifying the subset of patients at high risk of developing kidney disease, with a great impact on patients care.

The present study has investigated urine proteomic profile in adult BBS patients in comparison with age and sex-matched healthy volunteers. The results have been then stratified based on the eGFR, to address whether some classes of proteins correlated with kidney dysfunction. Finally, urine proteome of BBS patients has been correlated with retrospective data indicating the annual rate of eGFR decline.

The study shows that BBS patients have a particular urine proteome, differing from that of normal subjects. Fourtytwo proteins were deregulated in both male and female patients compared with control; the analysis of protein association networks revealed two networks consisting of 8 and 13 nodes respectively, extracellular matrix organization (ECM) and cell adhesion.

The association between ECM proteins and CKD has been already described in literature [33]. The putative underlying mechanism is the imbalance between ECM synthesis and degradation with subsequent fibrosis, a general pathophysiological mechanism in all types of kidney disease, independently of the initial injury [34]. Recently, Hijmans et al. have demonstrated that components of ECM proteins are detectable in the urine before the histological evidence of renal fibrosis, and have indicated urinary collagen degradation markers as potential diagnostic and predictive biomarkers of renal fibrosis [35]. In our study, ECM proteins showed different urinary abundance in BBS patients compared with controls, even in the absence of GFR decline. As all BBS patients had renal abnormalities on ultrasound, it is possible that this finding may indicate these molecules as early markers of kidney defects, that proceed GFR decline. Longitudinal studies, as well as metabolomic analysis [34], will enable us to address whether this data may predict the progression of 


\section{Kidney Blood Pressure Research}

Kidney Blood Press Res 2018;43:389-405

\begin{tabular}{l|l}
\hline DOI: $10.1159 / 000488096$ & (c) 2018 The Author(s). Published by S. Karger AG, Basel
\end{tabular}

Published online: March 13, 2018

www.karger.com/kb

renal failure.

CAMs are critical for either cell-cell and cell-extracellular matrix interactions [36]. We found significant differences in urinary abundance of cadherins and integrins in BBS patients. The meaning of this association remains to be elucidated. In the best studied experimental model of ciliopathy, the autosomal dominant polycystic kidney disease (ADPKD), it has been largely demonstrated the role of polycistin-1 (PC-1) in cell-matrix and cell-cell interactions [37]. Recently, Castelli et al. have shown that PC-1 overexpression in cells leads to enhanced adhesion to the substrate, while PC-1 downregulation reduced this ability via microtubule cytoskeleton regulation [38]. It is now clear that PC-1 has also extra-cilia localization, raising the hypothesis that some features of ADPKD are based on cilia-independent cellular defects. The role of BBS genes in biological functions other than PC homeostasis has been suggested by some studies. Tobin et al. demonstrated that BBS 8 knocking down in zebrafish caused defective neural crest migration [39]. Accordingly, Hernandez et al. have demonstrated that $B B S 4$ and BBS6 null cells showed deficient migration and adhesion abilities, disruption of actin stress fiber formation and focal adhesions [40], indicating a possible role of BBS genes in cytoskeleton organization and related biological processes. In a recent study we showed that $B B S 10$ silencing in a mouse cortical collecting duct-derived cell line led to a dramatic reduction of tubulin acetylation, associated with an impaired AQP2 trafficking to the apical membrane [9]. Whether other apical channels and/or transporters are affected remains to be elucidated $[41,42]$. The molecular mechanisms linking BBS genes to cytoskeleton organization and its role in the pathophysiology of BBS defects requires additional studies. We may speculate that cytoskeleton defects can cause aberration in ciliogenesis and signaling pathways, that eventually lead to kidney and other organs defects.

The analysis of urine proteome profile with respect to the eGFR demonstrated a significant increased abundance of fibronectin, CD44 antigen and lysosomal alfa glucosidase when the GFR declines. Fibronectin is a component of the extracellular matrix, whose deposition is believed to lead to glomerular and tubular basement membrane thickening and ultimately to glomerulosclerosis and interstitial fibrosis in almost all kidney diseases [43]. The correlation between its abundance and the eGFR strongly supports the hypothesis that it may reflect renal pathology.

CD44 antigen is a transmembrane protein undetectable or barely detectable in the normal kidney, that is expressed in the proximal tubule upon ischemic injury and in renal cell tumors [44]. In mice, it is expressed during T-cell mediated tubule-interstitial nephritis, and it is believed to play a role in cell repair through cell binding to the ECM [45]. Previous proteomics studies have identified the association between CD44 antigen and renal disease in several clinical conditions, including renal transplant rejection, IgA nephropathy and focal segmental glomeruloscherosis (FSGS) [46, 47].Our findings confirm that this protein correlates with renal dysfunction also in BBS. The increased urinary abundance of the lysosomal alfa glucosidase is more difficult to interpret. The protein has a crucial role in glycogen degradation to glucose, and its dysfunction due to mutations in the GAA gene leads to the glycogen storage disease type II (Pompe disease).The protein is largely expressed in the proximal tubule (PT). Whether the increased urinary abundance in patients with lower GFR is a generic marker of nephron disruption remains to be elucidated. The current data do not support a possible role of general or specific PT dysfunction in BBS patients, but further studies are needed to answer this questions [48].

A series of proteins, including vitronectin, Ig kappa chain V-III Isoform 2 and Tyrosineprotein phosphatase non-receptor type substrate 1 (SIRP- $\alpha)$ were significantly correlated with the eGFR separately in woman and men but not when we considered the whole cohort, maybe for the inter-gender variability $[49,50]$; it is not excluded that they may be part of a panel of proteins that reflect kidney dysfunction.

Finally, urine proteomes were analyzed based on mean annual eGFR decline. Interestingly, the majority of BBS patients showed a progressive decline of the eGFR, suggesting that those patients are at high risk to develop kidney failure. Whether intrinsic factors related 


\section{Kidney Blood Pressure Research}

to the genetic disease or common risk factors, as hypertension, diabetes and obesity may play a role in GFR decline is currently unknown and is object of intensive investigations. In our cohort there is a little presence of diabetic patients, and only 2 of 14 patients were hypertensive; surely these conditions may play a role in kidney disease progression, as in general population, but the evidence of GFR decline in non hypertensive and non diabetic patients suggests that other factors may participate in the pathogenesis of GFR decline. Larger studies are needed to address whether obesity may play a more significant role.

Interestingly, our data demonstrate a significant correlation between fibronectin and $\triangle \mathrm{eGFR}$. This finding further confirms the importance of this protein as a marker of kidney disease.

Besides the proteins previously described, a series of other proteins have been found over or under- represented in the urine of BBS patients compared with controls, that may be of potential interest in the understanding the pathogenesis of renal and extra renal dysfunction. Some of those proteins are surely of renal origin. It is the case of Uromodulin, whose abundance is reduced in female BBS patients, confirming our previous data [6]. The protein is kidney-specific, and is the most abundant tubular protein in normal urine [51, 52]. Recently, much attention has been paid on its urinary levels given the correlation with incident CKD and kidney disease progression [53]. Other proteins may derive from the blood, considering that whole urine samples have been used in our study. Even though we focused on kidney disease, some of our findings may shed light on extra-renal dysfunctions of BBS patients. Male BBS individuals showed a reduced urine excretion of the lysosomal enzyme N-acetylglucosamine-6-sulfatase. Protein deficiency results in the accumulation of under-degraded substrate, in mucopolysaccharidosis type IIID (Sanfilippo D syndrome), a clinical condition characterized by intellectual disability and behavioral disturbances [54]. Whether this finding may have a correlation with learning disabilities, a common feature of BBS patients, is currently unknown. In addition, both male and female BBS patients showed a reduced urine excretion of the Zinc-alpha-2-glycoprotein (ZAG). This protein is implicated in multiple biological processes, including lipid mobilization, cell cycling, tumor proliferation, and transport of nephritic by-products [55]. It has been recently recognized as a negative regulator of fibrosis progression [56], and our finding may indicate a role in renal and/or extra-renal disease in BBS.

We are aware that the current study has a number of limitations. First, pooled urine was used for proteomics analysis of the controls, and this clearly limited the interpretation of data. Second, whole urine samples have been used as source of proteins in both patients and controls, as stated above, while the recent literature has shown the advantages of using exosomes in studying renal diseases, as exosomes extraction reduces the complexity of urine and ensures the renal and urinary tract origin of the found molecules. In addition, due to sample preparation, low molecular weight proteins could have been missed, limiting the information on the whole proteome profile. Moreover, even though the number of patients with low eGFR is limited, the absence of a control group of (early stages) CKD subjects does not allow a firm conclusion on the meaning of over and under- represented proteins, as this effect may be the result of impaired renal function in general. Finally, even if the disease is rare, the number of patients is quite limited, and a larger study is required to confirm the current findings and to address whether additional pathways may be identified in the subset of patients with kidney dysfunction. To this purpose, including BBS patients with more advanced renal disease will be of help in understanding whether derangement of metabolic pathways common to CKD from any cause (as oxidative stress and sulfur aminoacid metabolism [57]) or disease-specific biological processes underlie renal disease progression and its complications [58].

However, given the rarity of the disease, the correlations we found may serve as a pilot study in the discovery of urinary markers of kidney dysfunction. 


\section{Kidney Blood Pressure Research}

\section{Conclusion}

Our data indicate the potentiality of urine proteomics in the discovery of early markers of eGFR decline in BBS. Larger prospective studies are required to confirm these findings. The possibility to assess the risk to develop chronic kidney disease will impact the management of BBS patients, enabling to start preventive strategies in time.

\section{Disclosure Statement}

All authors declared no competing interests.

\section{Acknowledgements}

This work was supported in part by a grant from the Italian Association of Bardet-Biedl Patients (ASBBI).

\section{References}

1 Álvarez-Satta M, Castro-Sánchez S, Valverde D: Bardet-Biedl Syndrome as a Chaperonopathy: Dissecting the Major Role of Chaperonin-Like BBS Proteins (BBS6-BBS10-BBS12). Front Mol Biosci 2017;4:55.

-2 Imhoff O, Marion V, Stoetzel C, Durand M, Holder M, Sigaudy S, Sarda P, Hamel CP, Brandt C, Dollfus H, Moulin B: Bardet-Biedl syndrome: a study of the renal a cardiovascular phenotypes in a French cohort. Clin J Am Soc Nephrol 2011;6:22-29.

-3 Esposito G, Testa F, Zacchia M, Crispo AA, Di Iorio V, Capolongo G, Rinaldi L, D’Antonio M, Fioretti T, Iadicicco P, Rossi S, Franzè A, Marciano E, Capasso G, Simonelli F, Salvatore F: Genetic characterization of Italian patients with Bardet-Biedl syndrome and correlation to ocular, renal and audio-vestibular phenotype: identification of eleven novel pathogenic sequence variants. BMC Med Genet 2017;18:10.

-4 Forsythe E, Sparks K, Best S, Borrows S, Hoskins B, Sabir A, Barrett T, Williams D, Mohammed S, Goldsmith D, Milford DV, Bockenhauer D, Foggensteiner L, Beales PL: Risk Factors for Severe Renal Disease in BardetBiedl Syndrome: J Am Soc Nephrol 2017;28:963-970.

-5 Beales PL, Elcioglu N, Woolf AS, Parker D, Flinter FA: New criteria for improved diagnosis of Bardet-Biedl syndrome: results of a population survey. J Med Genet 1999;36:437-446.

-6 Zacchia M, Zacchia E, Zona E, Capolongo G, Raiola I, Rinaldi L, Trepiccione F, Ingrosso D, Perna A, Di Iorio V, Simonelli F, Moe OW, Capasso G: Renal phenotype in Bardet-Biedl syndrome: a combined defect of urinary concentration and dilution is associated with defective urinary AQP2 and UMOD excretion. Am J Physiol Renal Physiol 2016;311:F686-F694.

-7 Harnett JD, Green JS, Cramer BC, Johnson G, Chafe L, McManamon P, Farid NR, Pryse-Phillips W, Parfrey PS: The spectrum of renal disease in Laurence-Moon-Biedl syndrome. N Engl J Med 1988;319:615-618.

8 Zona E, Zacchia M, Di Iorio V, Capolongo G, Rinaldi L, Capasso G: Patho-physiology of renal dysfunction in Bardet-Biedl Syndrome. G Ital Nefrol 2017;34:62-72.

-9 Zacchia M, Esposito G, Carmosino M, Zacchia E, Crispo AA, Fioretti T, Trepiccione F, Di Iorio V, Simonelli F, Salvatore F, Capasso G, Svelto M, Procino G: Knockdown of the BBS10 Gene Product Affects Apical Targeting of AQP2in Renal Cells: A Possible Explanation for the Polyuria Associated with Bardet-Biedl Syndrome. Genet Syndr Gene Ther 2014,5:3.

10 Lang F, Guelinckx I, Lemetais G, Melander O: Two Liters a Day Keep the Doctor Away? Considerations on the Pathophysiology of Suboptimal Fluid Intake in the Common Population. Kidney Blood Press Res 2017;42:483-494.

11 Zacchia M, Capasso G: Dehydration: A new modulator of klotho expression. Am J Physiol Renal Physiol 2011;301:F743-F744. 


\section{Kidney \\ Blood Pressure Research}

12 Zacchia M, Capolongo G, Rinaldi L, Capasso G: Renal handling of uric acid. G Ital Nefrol 2015;32:S62.

13 Zacchia M, Capolongo G, Trepiccione F, Marion V: Impact of local and systemic factors on kidney dysfunction in Bardet-Biedl Syndrome. Kidney Blood Press Res 2017;42:784-793.

14 Ansley SJ, Badano JL, Blacque OE Hill J, Hoskins BE, Leitch CC, Kim JC, Ross AJ, Eichers ER, Teslovich TM, Mah AK, Johnsen RC, Cavender JC, Lewis RA, Leroux MR, Beales PL, Katsanis N: Basal body dysfunction is a likely cause of pleiotropic Bardet-Biedl syndrome. Nature 2003;425:628-633.

15 Guo DF, Beyer AM, Yang B, Nishimura DY, Sheffield VC, Rahmouni K: Inactivation of Bardet-Biedl syndrome genes causes kidney defects. Am J Physiol Renal Physiol 2011;300:F574-F580.

-16 Cognard N, Scerbo Mj, Obringer C Yu X, Costa F, Haser E, Le D, Stoetzel C, Roux MJ, Moulin B, Dollfus H, Marion V: Comparing the Bbs10 complete knockout phenotype with a specific renal epithelial knockoutone highlights the link between renal defects and systemic inactivation in mice. Cilia 2015;4:10.

17 Marion V, Schlicht D, Mockel A, Caillard S, Imhoff O, Stoetzel C, van Dijk P, Brandt C, Moulin B, Dollfus H: Bardet-Biedl syndrome highlights the major role of the primary cilium in efficient water reabsorption. Kidney Int 2011;79:1013-1025.

18 Zacchia M, Di Iorio V, Trepiccione F, Caterino M, Capasso G: The Kidney in Bardet-Biedl Syndrome: Possible Pathogenesis of Urine Concentrating Defect. Kidney Dis (Basel) 2017;3:57-65.

19 Van JA, Scholey JW, Konvalinka A: Insights into Diabetic Kidney Disease Using Urinary Proteomics and Bioinformatics. J Am Soc Nephrol 2017;28:1050-1061.

20 Beretov J, Wasinger VC, Schwartz P, Graham PH, Li Y: A standardized and reproducible urine preparation protocol for cancer biomarkers discovery. Biomark Cancer 2014;6:21-27.

21 Caterino M, Pastore A, Strozziero MG, Di Giovamberardino G, Imperlini E, Scolamiero E, Ingenito L, Boenzi S, Ceravolo F, Martinelli D, Dionisi-Vici C, Ruoppolo M: The proteome of cblC defect: in vivo elucidation of altered cellular pathways in humans. J Inherit Metab Dis 2015,38:969-979.

-22 Caterino M, Chandler RJ, Sloan JL, Dorko K, Cusmano-Ozog K, Ingenito L, Strom SC, Imperlini E, Scolamiero E, Venditti CP, Ruoppolo M: The proteome of methylmalonic acidemia (MMA): the elucidation of altered pathways in patient livers. Mol Biosyst 2016,12:566-574.

23 Spaziani S, Imperlini E, Mancini A, Caterino M, Buono P, Orrù S: Insulin-like growth factor 1 receptor signaling induced by supraphysiological doses of IGF-1 in human peripheral blood lymphocytes. Proteomics 2014,14:1623-9.

24 Di Pasquale P, Caterino M, Di Somma A, Squillace M, Rossi E, Landini P, Iebba V, Schippa S, Papa R, Selan L, Artini M, Palamara AT, Duilio A: Exposure of E. coli to DNA-Methylating Agents Impairs Biofilm Formation and Invasion of Eukaryotic Cells via Down Regulation of the N-Acetylneuraminate Lyase NanA. Front Microbiol 2016;7:147.

25 Alberio T, Pieroni L, Ronci M, Banfi C, Bongarzone I, Bottoni P, Brioschi M, Caterino M, Chinello C, Cormio A, Cozzolino F, Cunsolo V, Fontana S, Garavaglia B, Giusti L, Greco V, Lucacchini A, Maffioli E, Magni F, Monteleone F, et al.: Toward the Standardization of Mitochondrial Proteomics: The Italian Mitochondrial Human Proteome Project Initiative. J Proteome Res 2017;16:4319-4329.

-26 Caterino M, Aspesi A, Pavesi E, Imperlini E, Pagnozzi D, Ingenito L, Santoro C, Dianzani I, Ruoppolo M: Analysis of the interactome of ribosomal protein 19 mutants. Proteomics 2014;14:2286-2296.

-27 Nigro E, Imperlini E, Scudiero O, Monaco ML, Polito R, Mazzarella G, Orrù S, Bianco A, Daniele A: Differentially expressed and activated proteins associated with non small cell lung cancer tissues. Respir Res 2015;24:16:74.

28 Zybailov B, Mosley AL, Sardiu ME, Coleman MK, Florens L, Washburn MP: Statistical analysis of membrane proteome expression changes in Saccharomyces cerevisiae. J Proteome Res 2006;5:2339-2347.

29 Capobianco V, Caterino M, Iaffaldano L, Nardelli C, Sirico A, Del Vecchio L, Martinelli P, Pastore L, Pucci P, Sacchetti L: Proteome analysis of human amniotic mesenchymal stem cells (hA-MSCs) reveals impaired antioxidant ability, cytoskeleton and metabolic functionality in maternal obesity. Sci Rep 2016;6:25270.

-30 Imperlini E, Santorelli L, Orrù S, Scolamiero E, Ruoppolo M, Caterino M: Mass Spectrometry-Based Metabolomic and Proteomic Strategies in Organic Acidemias. Biomed Res Int 2016;2016:9210408.

-31 Zacchia M, Vilasi A, Capasso A, Morelli F, De Vita F, Capasso G: Genomic and proteomic approaches to renal cell carcinoma. J Nephrol 2011;24:155-164.

32 Costanzo M, Zacchia M, Bruno G, Crisci D, Caterino M, Ruoppolo M: Integration of Proteomics and Metabolomics in Exploring Genetic and Rare Metabolic Diseases. Kidney Dis (Basel) 2017;3:66-77. 


\section{Kidney \\ Blood Pressure Research}

33 Mullen W, Deles C, Mischak H, EuroKUP COST action: Urinary proteomics in the assessment of chronic kidney disease. Curr Opin Nephrol Hypertens 2011;20:654-661.

-34 Scolamiero E, Cozzolino C, Albano L, Ansalone A, Caterino M, Corbo G, di Girolamo MG, Di Stefano C, Durante A, Franzese G, Franzese I, Gallo G, Giliberti P, Ingenito L, Ippolito G, Malamisura B, Mazzeo P, Norma A, Ombrone D, Parenti G, et al: Targeted metabolomics in the expanded newborn screening for inborn errors of metabolism. Mol Biosyst 2015;11:1525-1535.

-35 Hijmans RS, Rasmussen DG, Yazdani S Navis G, van Goor H, Karsdal MA, Genovese F, van den Born J: Urinary collagen degradation products as early markers of progressive renal fibrosis. J Transl Med 2017;15:63.

-36 Bays JL, DeMali KA: Vinculin in cell-cell and cell-matrix adhesions. Cell Mol Life Sci 2017;74:2999-3009.

-37 Wilson PD, Geng L, Li X, Burrow CR: The PKD1 gene product, "polycystin-1," is a tyrosine-phosphorylated protein that colocalizes with alpha2beta1-integrin in focal clusters in adherent renal epithelia. Lab Invest 1999;79:1311-1323.

-38 Castelli M, De Pascalis C, Distefano G, Ducano N, Oldani A, Lanzetti L, Boletta A: Regulation of the microtubular cytoskeleton by Polycystin-1 favors focal adhesions turnover to modulate cell adhesion and migration. BMC Cell Biol 2015;16:15.

39 Tobin JL, Di Franco M, Eichers E, May-Simera H, Garcia M, Yan J, Quinlan R, Justice MJ, Hennekam RC, Briscoe J, Tada M, Mayor R, Burns AJ, Lupski JR, Hammond P, Beales PL: Inhibition of neural crest migration underlies craniofacial dysmorphology and Hirschsprung's disease in Bardet-Biedl syndrome. Proc Natl AcadSci U S A 2008;105:6714-6719.

40 Hernandez-Hernandez V, Pravincumar P, Diaz-Font A, May-Simera H, Jenkins D, Knight M, Beales PL: Bardet-Biedl syndrome proteins control the cilia length through regulation of actin polymerization. Hum Mol Genet 2013;22:3858-3868.

41 Capasso G, Rizzo M, Garavaglia ML, Trepiccione F, Zacchia M, Mugione A, Ferrari P, Paulmichl M, Lang F, Loffing J, Carrel M, Damiano S, Wagner CA, Bianchi G, Meyer G: Upregulation of apical sodium-chloride cotransporter and basolateral chloride channels is responsible for the maintenance of salt-sensitive hypertension. Am J Physiol Renal Physiol 2008;295:F556-F567.

42 Petrazzuolo 0, Trepiccione F, Zacchia M, Capasso G: Hypertension and renal calcium transport. J Nephrol 2010;23:S112-S117.

43 Hu C, Sun L, Xiao L, Han Y, Fu X, Xiong X, Xu X, Liu Y, Yang S, Liu F, Kanwar YS: Insights into the Mechanisms Involved in the Expression and Regulation of Extracellular Matrix Proteins in Diabetic Nephropathy. Curr Med Chem 2015;22:2858-2870.

44 Lewington AJ1, Padanilam BJ, Martin DR, Hammerman MR: Expression of CD44 in kidney after acute ischemic injury in rats. Am J Physiol Regul Integr Comp Physiol 2000;278:R247-R254.

45 Naor D, Sionov RV, Ish-Shalom D: CD44: structure, function, and association with the malignant process. Adv Cancer Res 1999;71:241-319.

46 Sigdel TK, Kaushal A, Gritsenko M, Norbeck AD, Qian WJ, Xiao W, Camp DG 2nd, Smith RD, Sarwal MM: Shotgun proteomics identifies proteins specific for acute renal transplant rejection. Proteomics Clin Appl 2010;4:32-47.

-47 Prikryl P, Vojtova L, Maixnerova D, Vokurka M, Neprasova M, Zima T, Tesar V: Proteomics approach for identification of IgA nephropathy-related biomarkers in urine. Physiol Res 2017;66:621-632.

48 Trepiccione F, Zacchia M, Capasso G: Physiopathology of Potassium Deficiency; in Alpern RJ, Caplan MJ, Moe OW (eds): Seldin and Geibisch's The Kidney Physiology and Pathophysiology (Fifth Edition). Elsevier, 2013 pp 1713-1738.

49 Ruoppolo M, Campesi I, Scolamiero E, Pecce R, Caterino M, Cherchi S, Mercuro G, Tonolo G, Franconi F: Serum metabolomic profiles suggest influence of sex and oral contraceptive use. Am J Transl Res 2014;6:614-624.

50 Ruoppolo M, Scolamiero E, Caterino M, Mirisola V, Franconi F, Campesi I: Female and male human babies have distinct blood 1 metabolomic patterns. Mol Biosyst 2015;11:2483-2492.

51 Zacchia M, Capasso G: The importance of uromodulin as regulator of salt reabsorption along the thick ascending limb. Nephrol Dial Transplant 2015;30:158-160.

-52 Zacchia M, Capasso G: Parvalbumin: a key protein in early distal tubule $\mathrm{NaCl}$ reabsorption. Nephrol Dial Transplant 2008;23:1109-1111. 


\section{Kidney Research}

53 Devuyst 0, Bochud M: Uromodulin, kidney function, cardiovascular disease, and mortality. Kidney Int 2015;88:944-946.

-54 Beesley CE, Burke D, Jackson M, Vellodi A, Winchester BG, Young EP: Sanfilippo syndrome type D: identification of the first mutation in the N-acetylglucosamine-6-sulphatase gene. J Med Genet 2003;40:192-194.

-55 Hassan MI, Waheed A, Yadav S, Singh TP, Ahmad F: Zinc alpha 2-glycoprotein: a multidisciplinary protein. Mol Cancer Res 2008;6:892-906.

-56 Sörensen-Zender I, Bhayana S, Susnik N, Rolli V, Batkai S, Baisantry A, Bahram S, Sen P, Teng B, Lindner R, Schiffer M, Thum T, Melk A, Haller H, Schmitt R: Zinc- $\alpha 2$-Glycoprotein Exerts Antifibrotic Effects in Kidney and Heart. J Am Soc Nephrol 2015;26:2659-2668.

57 Perna AF, Zacchia M, Trepiccione F, Ingrosso D: The Sulfur Metabolite Lanthionine: Evidence for a Role as a Novel Uremic Toxin. Toxins (Basel) 2017, DOI: 10.3390/toxins9010026.

58 Capasso R, Sambri I, Cimmino A, Salemme S, Lombardi C, Acanfora F, Satta E, Puppione DL, Perna AF, Ingrosso D: Homocysteinylated albumin promotes increased monocyte-endothelial cell adhesion and upregulation of MCP1, Hsp60 and ADAM17. PLoS One 2012;7:e31388. 